SANDIA REPORT

SAND98-0704 • UC-426

Unlimited Release

Printed May 1998
AECEIVED

JUN $O 31998$

OSTI

Design and Experimental Results on a Terawatt Magnetically Controlled Plasma Opening Switch

DISTRIBUTION OF THIS DOCUMENT IS UNLMITED

M. E. Savage, W. W. Simpson, C. W. Mendel, Jr., D. H. McDaniel, J. S. Levine, T. S. Tucker

Prepared by

Sandia National Laboratories

Albuquerque, New Mexico 87185 and Livermore, California 94550

Sandia is a multiprogram laboratory operated by Sandia Corporation, a Lockheed Martin Company, for the United States Department of

Energy under Contract DE-AC04-94AL85000.

Approved for public release; further dissemination unlimited. 
Issued by Sandia National Laboratories, operated for the United States Department of Energy by Sandia Corporation.

NOTICE: This report was prepared as an account of work sponsored by an agency of the United States Government. Neither the United States Government nor any agency thereof, nor any of their employees, nor any of their contractors, subcontractors, or their employees, makes any warranty, express or implied, or assumes any legal liability or responsibility for the accuracy, completeness, or usefulness of any information. apparatus, product, or process disclosed, or represents that its use would not infringe privately owned rights. Reference herein to any specific commercial product, process, or service by trade name, trademark, manufacturer, or otherwise, does not necessarily constitute or imply its endorsement, recommendation. or favoring by the United States Government, any agency thereof, or any of their contractors or subcontractors. The views and opinions expressed herein do not necessarily state or reflect those of the United States Government, any agency thereof, or any of their contractors.

Printed in the United States of America. This report has been reproduced directly from the best available copy.

Available to DOE and DOE contractors from

Office of Scientific and Technical Information

P.O. Box 62

Oak Ridge, TN 37831

Prices available from (615) 576-8401, FTS 626-8401

Available to the public from

National Technical Information Service

U.S. Department of Commerce

5285 Port Royal Rd

Springfield, VA 22161

NTIS price codes

Printed copy: A04

Microfiche copy: A01

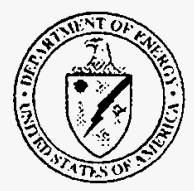




\title{
Design and experimental results on a terawatt magnetically controlled plasma opening switch
}

\author{
M. E. Savage, W. W. Simpson, C. W. Mendel, Jr., D. H. McDaniel \\ Accelerator Applications Department \\ Sandia National Laboratories \\ PO Box 5800 \\ Albuquerque, New Mexico 87185-1194 \\ J. S. Levine, T. S. Tucker \\ Primex Physics International \\ San Leandro, California 94577
}

\begin{abstract}
The magnetically controlled plasma opening switch (MCPOS) is an advanced plasma opening switch that utilizes magnetic fields to improve operation. Magnetic fields always dominate terawatt, pulsed power plasma opening switches. For that reason, the MCPOS uses controlled applied magnetic fields with magnitude comparable to the self-magnetic field of the storage inductor. One applied field holds the plasma in place while energy accumulates in the storage inductor, then another applied field pushes the plasma away from the cathode to allow energy to flow downstream.

Over a ten month period, an MCPOS was designed, built, and tested on DECADE Module 2 at Physics International. The peak drive current was $1.8 \mathrm{MA}$ in $250 \mathrm{~ns}$. The output parameters were up to $1 \mathrm{MA}$ into an electron beam load. The radiation temporal pulse width averaged 60 nanoseconds full-width at half-maximum. The peak load voltage ranged from one to two megavolts. The experiments demonstrated efficient power flow through a long, low-impedance magnetically insulated transmission line between the magnetically controlled plasma opening switch and the load.
\end{abstract}




\section{Acknowledgments}

These experiments would not have been possible without the talent of many people. John Maenchen helped with the DSWA collaboration, and provided many helpful comments on this manuscript. Andy Seth did an outstanding job of mechanical design of the parts on a short schedule. Richard Adler helped coordinate this operation with Physics International and DSWA, and suggested the machine shop that built the components. Jim Payne did an excellent job machining the complicated parts that went into the MCPOS. Bill Stygar volunteered helpful advice on building the applied magnetic field coils. Dave Seidel rapidly did computer modeling of the electron flow results, and helped us solve a problem with a single hardware modification. Larry Sanders was truly tireless in helping field the experiments on DM-2. Larry Francisco and the rest of the PI machine shop did outstanding machining and fabrications on short schedules. Physics International as a whole did an excellent job in the design and assembly of DM-2. Robin Sharpe was invaluable in supporting the Tesla experiments at Sandia. Don Cook provided managerial and monetary support, both of which are always appreciated. 


\section{Contents}

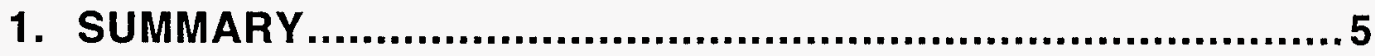

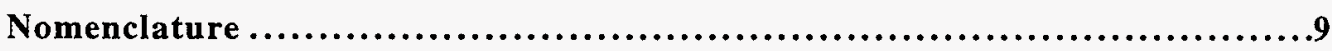

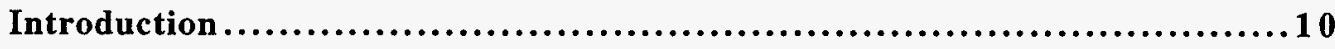

2. OPERATION............................................................ 13

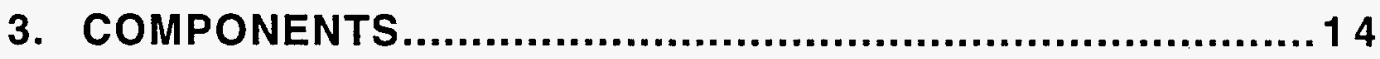

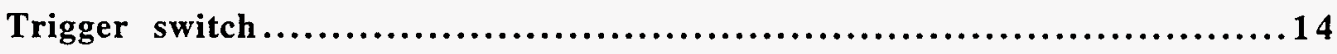

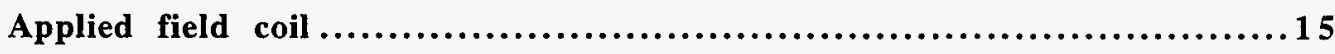

Main switch plasma source.......................................... 16

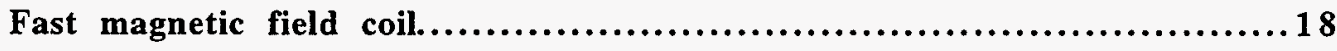

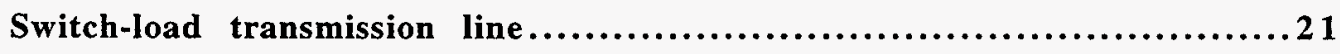

Electron beam load..................................................2 2

4. THE DM-2 EXPERIMENT AND DIAGNOSTICS ................24

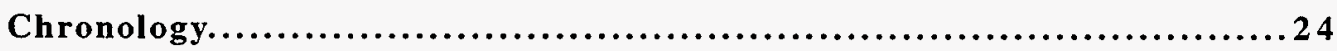

Installation.......................................................... 25

Diagnostics...........................................................2 6

Voltage measurements in magnetically insulated transmission lines.......28

Current monitors in the DM-2 system................................. 31

Metrics of opening switch performance................................ 32

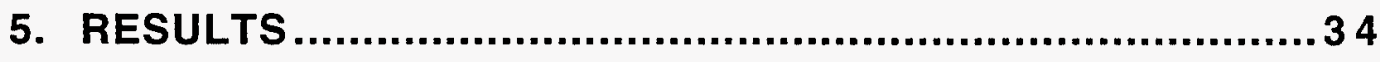

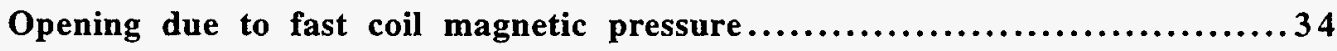

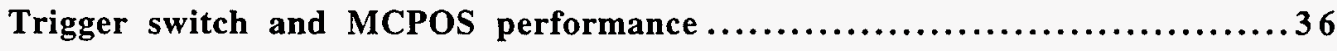

Load design and performance.........................................4 48 
Radiation production and measurements ...............................49

6. DISCUSSION ........................................................ 52

Voltage monitors in the DM-2 system.................................. 52

7. CONCLUSIONS................................................... 57

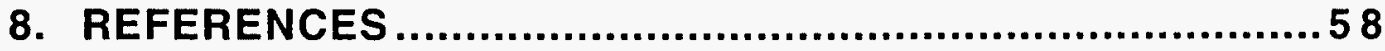

\section{Figures}

FIGURE 1-1. DECADE MODULE TWO. $\quad 7$

FIGURE 1-2. THE MAGNETICALLY CONTROLLED PLASMA OPENING SWITCH.

FIGURE 3-1 THE DM-2 MCPOS AND TRIGGER SWITCH HARDWARE 14

FIGURE 3-2. THE TRIGGER SWITCH REGION OF THE DM-2 SWITCH.

FIGURE 3-3. A DM-2 FLASHBOARD. 17

FIGURE 3-4. THE SWITCH, TRANSMISSION LINE, AND LOAD ON DM-2. 22

FIGURE 3-5. THE LOAD REGION OF DM-2. 23

FIGURE 4-1. GENERAL TRANSMISSION LINE MODEL. 29

FIGURE 4-2. OUTPUT OF THE INTEGRATORS USED ON DM-.

FIGURE 5-1. CURRENT MEASUREMENTS ON A DM-2 TEST (NORMAL APPLIED FIELD). 34

FIGURE 5-2. CURRENT MEASUREMENTS ON A DM-2 TEST (REVERSED APPLIED FIELD). 35

FIGURE 5-3. TRIGGER SWITCH AND MAIN SWITCH FLOW IMPEDANCE. 37

FIGURE 5-4. CALCULATED EFFECTIVE TRANSMISSION LINE GAP IN POS REGION. 38

FIGURE 5-5. CURRENTS ON DM-2.

FIGURE 5-6. CALCULATED VOLTAGES THROUGHOUT DM-2.

FIGURE 5-7. DM-2 MAIN SWITCH OPENING CURRENT.

FIGURE 5-8. DM-2 MAIN SWITCH OPENING CURRENT.

FIGURE 5-9. TRIGGER SWITCH FLOW IMPEDANCE.

FIGURE 5-10. SYSTEM FLOW IMPEDANCE.

FIGURE 5-11. THE RATIO OF THE FLOW IMPEDANCE OF THE MAIN SWITCH TO THE FLOW

IMPEDANCE OF THE TRIGGER SWITCH.

FIGURE 5-12. THE FLOW IMPEDANCE OF THE MAIN SWITCH VS THE RATIO OF APPLIED

AXIAL FIELD TO AXIAL FAST FIELD. $\quad 46$

FIGURE 5-13. THE FLOW IMPEDANCE OF THE MAIN SWITCH ON A SINGLE DM-2 TEST,

VERSUS THE RATIO OF APPLIED TO FAST FIELD $\quad 47$

FIGURE 5-14. LOAD IMPEDANCE ON DM-2 SHOT 243.

FIGURE 5-15. X-RAY PRODUCTION INFERRED FROM ELECTRICAL DIAGNOSTICS, AND XRAYS MEASURED BY THE FAR-FIELD PIN DIODE. 51

FIGURE 6-1. LOAD VOLTAGE.

FIGURE 6-2. OPENING SWITCH VOLTAGE. 53

FIGURE 6-3. PEAK VOLTAGE ON THE PIN DIODE SIGNAL VS. PEAK OF THE ELECTRICAL LOAD VOLTAGE. $\quad 54$

FIGURE 6-4. THE ELECTRICAL LOAD VOLTAGE COMPARED TO A LOAD VOLTAGE CALCULATED FROM THE PIN DIODE. 


\section{Summary}

The Defense Special Weapons Agency (DSWA) is developing a radiation effects testing simulator called DECADE. ${ }^{1} \mathrm{DECADE}$ is planned to be a large-area pulsed X-ray source, capable of delivering 20 kilorads in silicon over one square meter in 40 nanoseconds, with a X-ray spectral endpoint of $1.6 \mathrm{MeV}$. The design consists of 16 independent pulsers, each driving an electron-beam bremsstrahlung diode with a peak current of about 1 MA. Each module (Figure 1-1) has a low-inductance Marx generator, a water intermediate-storage capacitor, triggered gas closing switches, and a vacuum storage inductor. The vacuum storage inductor is terminated by a plasma opening switch, followed by the electron beam load. The opening switch remains closed while current rises and stores energy upstream of the opening switch. After about $250 \mathrm{~ns}$, when inductor current is about 1.8 MA, the vacuum opening switch should open, and quickly transfer energy into the electron beam load with significant power multiplication. This report details a magnetically controlled plasma opening switch (MCPOS) developed for this application, and tested on a prototype DECADE module, called DM-2.

Fast pulsed power, as studied at several laboratories over the past 30 years or more, is an application of electrical energy compression. Electrical energy accumulated over many seconds is compressed into shorter and shorter time intervals, and ultimately released in nanoseconds. This compression raises the power in inverse proportion to the time of release. Terawatt pulses ( $10^{12}$ watts) are now relatively common in the laboratory, even though this power level is comparable to the sustained power generation capacity of North America. The energy released in such pulses is usually of the order of tens to hundreds of kilojoules.

Such pulses, if converted to X-radiation, can mimic aspects of a nuclear explosion. This is one of the major application of terawatt pulses, to test the response of systems and components to the radiation flash of a nuclear explosion. This testing is more important now, because of the ban on nuclear detonations. The national need for Nuclear Weapons Effects Testing (NWET) must be met with sources other than underground nuclear explosions. One such source is DECADE, designed to be a general-purpose source of megavolt-endpoint X-radiation for Department of Defense and Department of Energy testing needs.

The DECADE system would require a total load power of $20 \mathrm{TW}$; each of the 16 independent pulsers would need to supply slightly over $1 \mathrm{TW}$. DECADE would use Inductive Energy Storage and a plasma opening switch for its final stage of pulse compression. This would make DECADE one of the largest inductive energy storage systems in use. However, there were serious difficulties with the conventional opening switch in initial testing, and there was interest in pursuing a second type of plasma opening switch. 
Towards that end, in 1995 and 1996, Sandia National Laboratories worked with Defense Special Weapons Agency (DSWA) and its contractors to test an alternate plasma opening switch on a prototype DECADE Module, called DM-2, at Physics International in California. This project would benefit both Sandia and DSWA, by testing the magnetically controlled plasma opening switch (MCPOS) in a substantially different regime than the previous successful MCPOS experiments. In those experiments it was proven that a plasma opening switch could be opened with the application of a fast magnetic field, and that such a switch would perform better than a conventional(inertial) plasma opening switch on the same pulser. The MCPOS is more complicated than conventional plasma opening switches, which simply launch plasma into a MITL gap, and rely upon inertia to keep the switch closed and drive current pressure to open the switch. Given the problems of some previous plasma opening switch programs, it is not surprising that demanding opening switch applications (long conduction time or high output power) would require sophisticated opening switches.

For the DM-2 experiment, the current switched by the MCPOS increased by a factor of two. The previous experiments were limited to $750 \mathrm{kA}$ peak current by the driver. Also, the DECADE system operates with a negative center conductor; the previous MCPOS experiments used a positive center conductor. The higher current and smaller cathode increased the cathode magnetic field (the important parameter) by a factor of three. This would test the understanding of the MCPOS. Such a large scale-up could not be expected to work without a valid model for the physics of the opening switch.

The MCPOS on DM-2 worked exactly as expected, proving the physics understanding of that switch. Unfortunately, the trigger switch, which energizes the magnetic field that opens the MCPOS performed poorly on DM-2, which limited the performance of the MCPOS. The trigger switch on DM-2 was an inertial POS because there was insufficient time to develop a better trigger switch. The MCPOS is essentially a power amplifier for the trigger switch. The MCPOS performed its action as expected, but the output of the trigger switch was a factor of 4 lower than needed, and that limited the main switch output. The important part of the test was a complete success. The trigger switch needs some development effort to raise its performance, and realize the potential of the MCPOS. The MCPOS and trigger switch as a system is interesting because of the power gain of the MCPOS. Reliable power amplifiers (like the MCPOS) are important in pulsed power. These experiments prove that small advances in the trigger switch will raise load power significantly, and that the MCPOS could be triggered with application of a fast field (which could be done with an external pulser, for example).

$\mathrm{X}$-radiation is an important measure of DECADE experiments. The goal for these experiments is a dose output that would be consistent with 20 kilorads (silicon) over 1 square meter for all 16 modules. The poor trigger switch performance limited the measured dose with the MCPOS to 12 kilorads equivalent. There was a total of ten X-ray production experiments (i.e., with a tantalum anode). Conservative scaling of the other 129 experiments showed some would have given about 18 kilorads had those tests used a tantalum converter. 
Figure 1-2 shows the MCPOS system, the switch-load transmission line, and the load installed on DECADE Module Two at PI. This report will show the design of the MCPOS system, and detail the driving physics concerns in such a design. This report will show the results of the experiment in general, and separate the performances of the MCPOS and trigger switches.

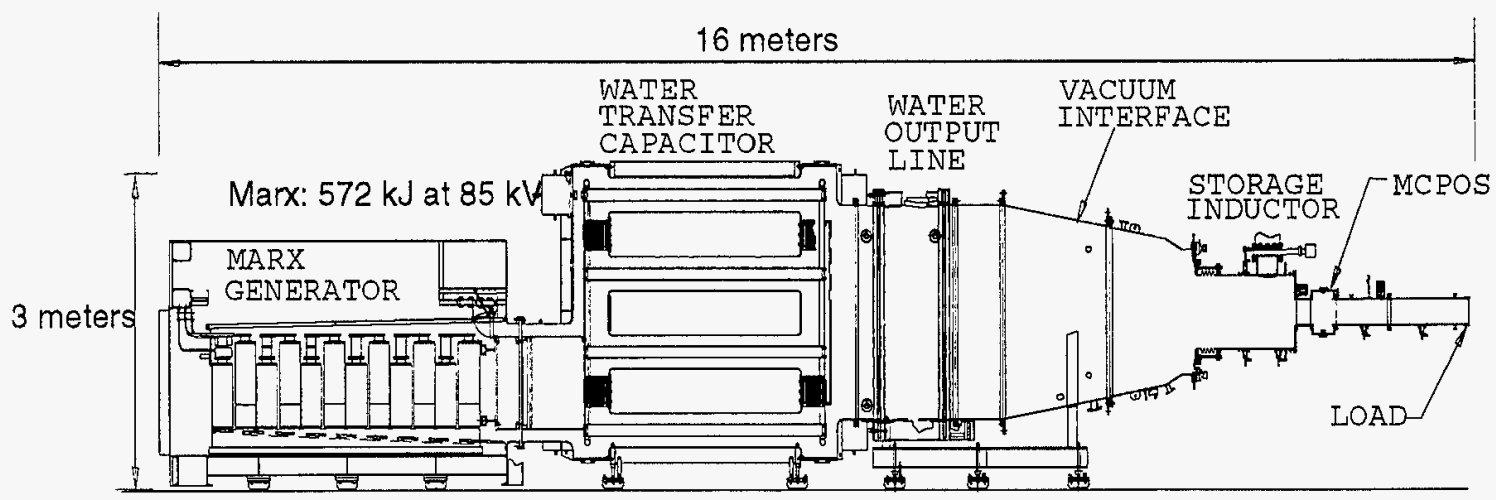

Figure 1-1. DECADE Module Two. The water transfer capacitor is switched into the water output line with six parallel triggered gas switches. The water output line is un-switched. 


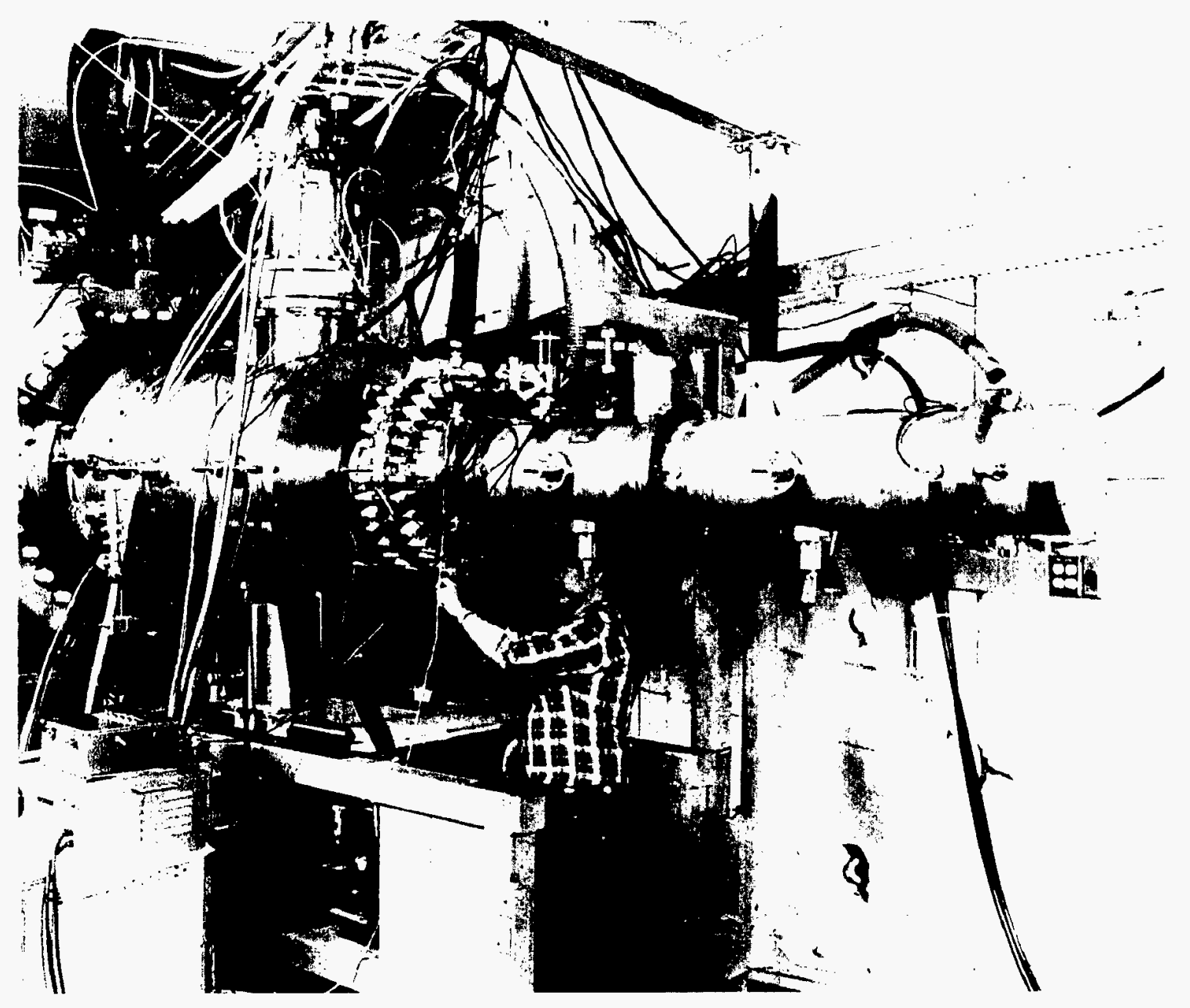

Figure 1-2. The magnetically controlled plasma opening switch installed on DECADE Module Two at Physics International. 


\section{Nomenclature}

Plasma opening switch (POS): a power compression device that provides a lowimpedance shunt across a vacuum transmission line gap, which opens after magnetic energy is stored in the upstream inductance. The load power is proportional to load impedance. Opening switches operate by moving an armature. Opening speed is determined by armature mass; for microsecond or faster time scales a plasma is the only choice for the armature because a plasma has both high conductivity and low mass. Opening switch power gain is accomplished by raising voltage across the load.

Magnetically controlled plasma opening switch (MCPOS): a plasma opening switch that uses magnetic fields to inject, and hold plasma in place while energy accumulates in the storage inductor. A second magnetic field actively pushes the plasma out of the transmission line gap, allowing energy to flow downstream.

Magnetically insulated transmission line (MITL): an electrical transmission line with vacuum dielectric. The self-magnetic field of load current flow suppresses electron loss at megavolt potentials.

Electron beam diode: a two-electrode high-voltage device to convert electrical energy into electron kinetic energy. The electron energy is often converted to X-rays with a high atomic number metal anode.

Magnetic field separatrix: coaxial coils with current in opposite directions (magnetic field between coils in the same axial direction) can have a zero flux surface between the coils, depending upon the geometry and relative currents in the coils. The surface essentially bounds each coils' flux. This surface will exist if the coils produce comparable magnetic fields, and their currents are in opposite direction.

Flow impedance: a relationship between voltage, anode current, and cathode current in a Magnetically Insulated Transmission Line. At megavolt potentials, flow impedance can range from slightly below the vacuum impedance, to a small fraction of the vacuum impedance. A vacuum opening switch often lowers the operating flow impedance downstream of the switch. At a given voltage and anode current, lower cathode current yields lower flow impedance.

Inductive energy storage systems: systems which store energy in magnetic field. The energy density is limited by mechanical strength rather than dielectric breakdown, so the energy density can be much higher than electrostatic storage. Because a magnetic field cannot impart energy to particles, avalanche breakdown (which limits the energy density of electrostatic storage systems) is impossible in an inductive energy store. Such systems use an opening switch to transfer energy into a load. 


\section{Introduction}

Inductive energy storage has advantages because of the high energy density possible. Inductive energy storage coupled with a vacuum opening switch has the added advantage of reducing the stress on the vacuum interface, which is the weakest link in the power flow chain. There are several kinds of vacuum opening switches in use, ${ }^{2-4}$ but the plasma opening switch is the most common for systems with moderate current densities ( 0.1 to 5 megamps per meter) and multi-megavolt voltages.

The plasma opening switch was invented in 1976,5 and was first used for eliminating pre-pulse on the Proto-I pulser, and was proposed at that time for pulsed power inductive energy storage systems. Since then, there has been a large effort to raise the energy stored in self-magnetically insulated transmission line (MITL) inductors upstream of the plasma opening switch, and to raise the ratio of load power to driver power. $6-8$ The advantages of inductive energy storage are well known, but the high power density that makes inductive energy storage attractive places severe demands on the opening switch. An opening switch requires at least an order of magnitude less volume than a comparable gas closing switch, offering the potential of substantially lower inductance, faster risetimes and higher power. The high electric field in the low-impedance regions of a POS makes axial electron flow important to power flow within and downstream of the plasma opening switch. Early opening switch research generally ignored electron flow in and downstream of the switch region, with the exception of Mendel ${ }^{5}$, Stringfield, ${ }^{9}$ and the notably thorough work of Bluhm ${ }^{10}$, each of whom measured cathode current downstream of the opening switch. Bluhm's observation in 1987 that power flow is affected by "remnant plasmas in the switch area" is among the first references to enhanced electron flow downstream of a plasma opening switch. Considerable theoretical and experimental work on magnetically insulated transmission lines $11-16$ also contributed to electron flow understanding. General electron flow considerations were applied to plasma opening switch experimental results at Sandia in 1987,17 and later at the Naval Research Laboratory and Maxwell Laboratories. 18-20 This electron flow theory has formed a common basis for comparing opening switches, emphasizing the launching and axial flowing of electrons as global characteristics. This is an alternative to modeling the switch as a radial resistor, which is load and geometry dependent, and therefore cannot generally be scaled between different systems. The electron flow approach place more emphasis on the launching and axial flowing of electrons, and less emphasis on the point at which electrons hit the anode. $21-23$ In an efficient system, electron flow is predominately axial, and therefore electrons may travel a considerable distance before reaching the anode. Electron flow measurements are vital in understanding opening switch performance and vacuum power flow, and this will be seen to be an integral part of these experiments.

There are two basic approaches to plasma opening switch research: passive and active. Passive techniques (demonstrated by the inertial plasma opening switch) rely on plasma inertia to keep the POS closed while energy accumulates in the inductor. Such systems necessarily open slower and less completely with longer conduction time. Active techniques (demonstrated by the magnetically controlled plasma opening switch) use an 
independent mechanism to initiate (trigger) opening. The majority of experiments and numerical efforts has been directed towards passive (inertial) plasma opening switches: for example, changes to switch length, switch electrode diameter, 24 plasma ion species, and electrode shapes. 25,26 The passive switch improvement techniques have always been empirical.

A smaller effort has been directed towards active switch improvement techniques: the Current Toggled plasma opening switch, 27 and the magnetically controlled plasma opening switch. 28 These active techniques use an applied field to inject and hold plasma in place during conduction, then use an additional magnetic field to push the conducting plasma out of the switch region during opening. The first experiments were the Current Toggle plasma opening switch. This opening switch employed a fast coil energized by load cathode current. This fast magnetic field coil applied a radial force to the plasma armature, moving the plasma armature. Subsequent experiments (the MCPOS) used a fast coil driven by pulser current, and controlled by a second plasma opening switch. The initial interest in this method of fast coil activation was to demonstrate active triggering of an opening switch. During those experiments, it was also observed that driving the fast field coil with driver current made the switch more tolerant of high impedance loads. This is important, because in an opening switch system, load power is higher with higher impedance loads.

The more mechanically complex active techniques are interesting only if they produce significant performance advantages over passive techniques. Active systems, as described here, have an inherent advantage because the opening mechanism (the fast magnetic field) is not related to the mechanisms that keeps the switch closed (plasma inertia and the applied magnetic field). The goal of the present experiments, and the previous experiments leading up to them, is an opening switch system that both works well and has an understandable physics model. This allows a robust linkage from hardware design to switch performance, avoiding costly and time-consuming full-power empirical optimization techniques used with passive systems. A valid model allows changing parameters on a design with predictable results. There is a large benefit to be had from a system that can be engineered and scaled. Possible advantages of active techniques are reduced development time and lower total project cost, higher electrical performance, and increased reproducibility. Simplified design and analysis is possible due to the increased role of magnetic fields, and the decreased role of plasma parameters. Sandia has spent all its opening switch development effort on active switches for the past 8 years.

The magnetically controlled plasma opening switch is an engineered device. The DM-2 experiments changed many parameters from the tests on Tesla at Sandia from 1990 to $1994.28,29$ These changes included raising the conduction current cathode magnetic field (the important current parameter) by a factor of three. The Tesla tests were in the opposite polarity of DM-2, and the total pulse energy was about a factor of three higher on DM-2. With similar (or smaller) extrapolations, other experiments have needed long series of trial-and-error testing, often without reaching design parameters. The understanding that the CTPOS and MCPOS adds is a quality that has been missing from opening switches before. The MCPOS is different for simple reasons: in the MCPOS design magnetic 
pressure dominates plasma pressure. This allows design with much reduced impact of plasma fill conditions and electrode plasmas. Reducing the effects of these parameters (which are much more difficult to control than magnetic fields) makes designing a switch tractable. 


\section{Operation}

MCPOS operation begins with energizing the applied field coil, which is a relatively low-voltage and slow ( $1 \mathrm{msec}$ ) coil. Within a few microseconds of peak applied field, the trigger POS and main POS plasma sources are triggered. The plasma source consists of 360 sparks on a $40 \mathrm{~cm}$ diameter. Plasma from individual sparks overlap about $1 \mathrm{~cm}$ from the flash board. At that distance magnetic pressure dominates plasma pressure, so the applied field guides the main switch plasma along the curved field lines into the magnetically insulated transmission line (MITL) anode-cathode gap. This plasma connects the anode and cathode of the MITL, and allows current to rise in the storage inductor.

The trigger switch, by contrast, is a conventional inertial POS with plasma injected radially, directly into the trigger POS gap, with an independent plasma source pulser. The trigger switch plasma short-circuits the fast magnetic field coil. When MITL current upstream of the MCPOS begins, the applied field is at its peak, and there is plasma in the trigger switch region and plasma in the main switch region. The plasma in the main switch short-circuits the MITL. The applied field, and plasma inertia keep the MCPOS plasma in place and the MITL shorted. As the vacuum inductor is charged, all driver current goes through the trigger switch. At a time close to peak driver current, the inertial trigger POS begins to open due to magnetic pressure and plasma thinning. As the trigger switch slowly opens, driver current begins to flow through the fast field coil, and then through the main switch plasma. The degree to which the trigger switch opens determines the fraction of upstream cathode current in the fast field coil. The fast magnetic field created by driver current flowing in the fast magnetic field coil pushes the trapped main switch plasma away from the cathode, actively opening the main switch. The axial field due to driver current is zero while the trigger POS is closed, and rises to several times the magnitude of the azimuthal driver field when the trigger POS opens. After the main switch opens, load current goes through the fast coil. If the trigger POS re-closes, flux will be trapped in the fast coil circuit. In either case, the MCPOS, once opened, will remain open until the vacuum inductor fully discharges into the load. 


\section{Components}

The major components of the MCPOS are the trigger plasma opening switch, the applied field coil, the main switch plasma source, and the fast field coil. Figure 3-1 shows a cross-section of the DM-2 magnetically controlled plasma opening switch and trigger switch.

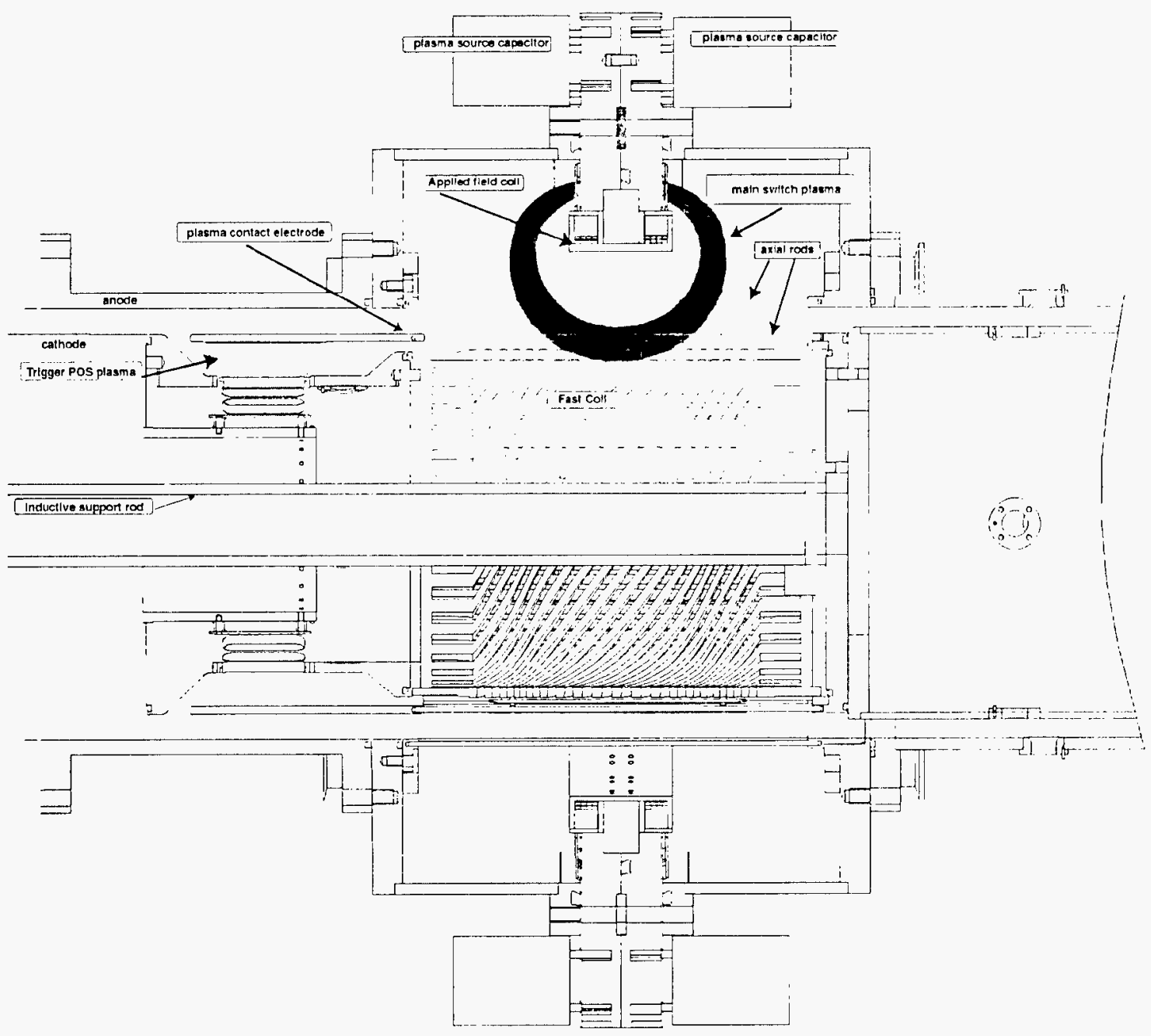

Figure 3-1 The DM-2 MCPOS and trigger switch hardware. The DM-2 drive pulse enters from the left; output power is delivered the load to the right. The fast coil diameter is $23 \mathrm{~cm}$. The indicated plasma fill shape is taken from magnetostatic flux contours without fast magnetic field.

\section{Trigger switch}

The trigger plasma opening switch, shown in Figure 3-2, is a conventional inertial opening switch with 20 flashboards. The flashboards are driven from a $10 \mathrm{kV}$, DCcharged capacitor and triggered spark gap in vacuum inside the DM-2 transmission line center conductor. Each flashboard connects to a $50 \mathrm{nF}, 10 \mathrm{kV}$ pulsed-charged capacitor 
through $50 \mathrm{~cm}$ of $10 \mathrm{ohm}$ coaxial cable. This provided a relatively low-inductance driver for the trigger POS flashboards, though it was much higher inductance than the main plasma source system. The plasma drift distance is $2 \mathrm{~cm}$, and the switch anode-cathode gap is $2 \mathrm{~cm}$. The cathode of the trigger switch is connected to the upstream DM-2 cathode. The anode of the trigger switch is the Plasma Contact Electrode, which connects to the downstream end of the fast coil. In the trigger switch region, the Plasma Contact Electrode is a tube. In the MCPOS region, the Plasma Contact Electrode is an array of 36 axial rods, to allow fast magnetic field into the MITL gap. The trigger switch is electrically across the fast field coil, and a closed trigger switch short-circuits the fast coil. When the trigger switch opens, current flows in the fast coil. After the main switch opens, the current flows through the fast coil and through the main switch plasma. If the main switch is open, fast coil current flows to the load. The electric field on the fast coil surface is due to trigger switch voltage and not the much higher main switch voltage. Energetic ions at MITL potential strike the Plasma Contact Electrode, and not the fast field coil. The MCPOS cathode is the plasma contact electrode, and not the fast coil. Electrons launched in the main switch region will come from the plasma contact electrode, and therefore this current must flow in the fast coil and contribute to axial field.

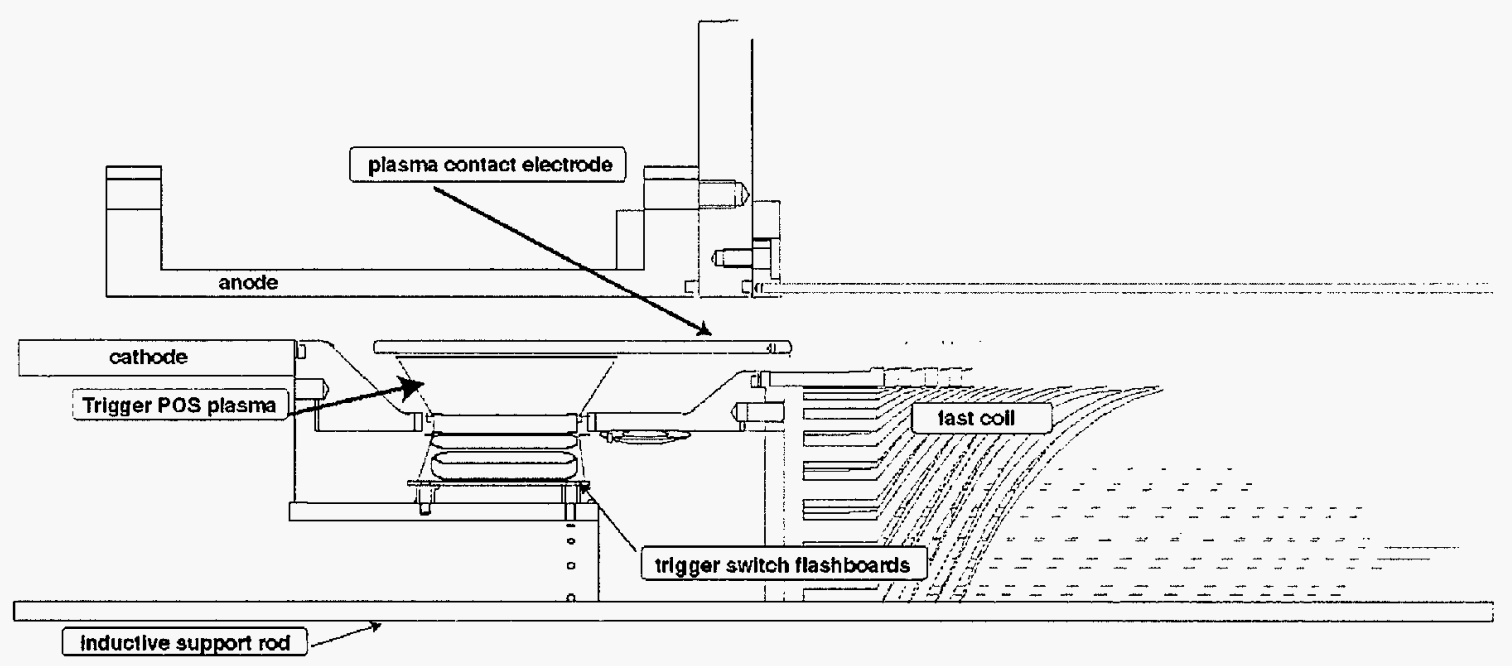

Figure 3-2. The trigger switch region of the DM-2 switch. The plasma contact electrode connects to the downstream end of the fast coil. The monitor for the fast coil current is about $2 \mathrm{~cm}$ past the downstream end of the trigger switch plasma region, on the cathode side.

\section{Applied field coil}

The applied field coil is a moderate voltage $(10 \mathrm{kV})$, multi-turn coil. The coil's efficiency is determined by the resistance of the windings, and the resistivity of nearby conductors. Neglecting external flux penetration, the efficiency (ratio of magnetic energy at peak current divided by capacitive stored energy) of a coil in an underdamped RLC circuit is: 


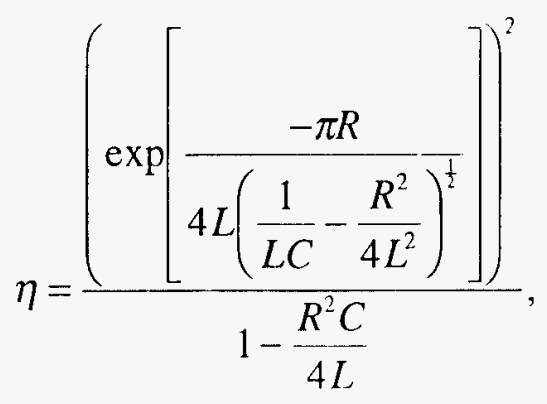

where $\mathrm{R}$ is the system resistance, $\mathrm{C}$ is the bank capacitance, and $\mathrm{L}$ is the total inductance. The efficiency equation above implies $0.15 \Omega$ total resistance on the tests at Sandia; of the total, $0.04 \Omega$ is attributable to the coil. The DM-2 applied field system operated at 30 percent efficiency with the same coil and a $240 \mathrm{~kJ}, 10 \mathrm{kV}$ bank.

The MCPOS is designed with some components through which magnetic field penetration is desirable (the metal coil form for example), and some picces the field should not penetrate (field-shaping flux excluders). Titanium-alloy and stainless steel are used for the former, and oxygen-free copper for the flux excluders. Mechanical engincering constrains the minimum thickness of the coil form, which restricts quarter-period times to greater than a few hundred $\mu \mathrm{s}$. Thus, for multi-hundred kilojoule, $10 \mathrm{kV}$ capacitor banks, a one to two hundred $\mu \mathrm{H}$ coil is desirable. The 16 turn DM-2 applied field coil mects this inductance requirement, with a time to peak current of one millisecond. This allowed low losses with penetration of 3-8 mm of stainless stecl. The flux excluders were several times thicker than the $4.2 \mathrm{~mm}$ skin depth in copper.

\section{Main switch plasma source}

The MCPOS main switch plasma source must supply adequate plasma to conduct the DM-2 current pulse. The plasma fill should be azimuthally uniform and hollow, as indicated in Figure 3-1. The plasma should also be axially centered within the fast coil to be acted upon by the fast field. The uniformity requirement comes about because the plasma must provide an azimuthal current path to exclude axial fast magnetic field. A hollow fill minimizes the total plasma mass that must be moved. Experiments with solidplasma fill configurations at Sandia at 220 ns conduction times showed less complete opening, as would be expected from excessive mass. The plasma density on this source has not been measured, but is expected to be a few times $10^{14}$ electrons per $\mathrm{cm}^{3}$ in the switch region, based on measurements done on a similar source. 27

Figure 3-3 shows a flashboard and a conceptual sketch of plasma expansion from a board. The conceptual sketch indicates plasma at about $100 \mathrm{~ns}$ after flashboard current begins. 

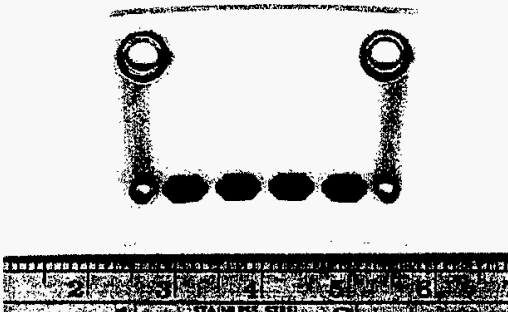

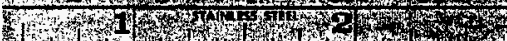

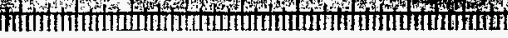

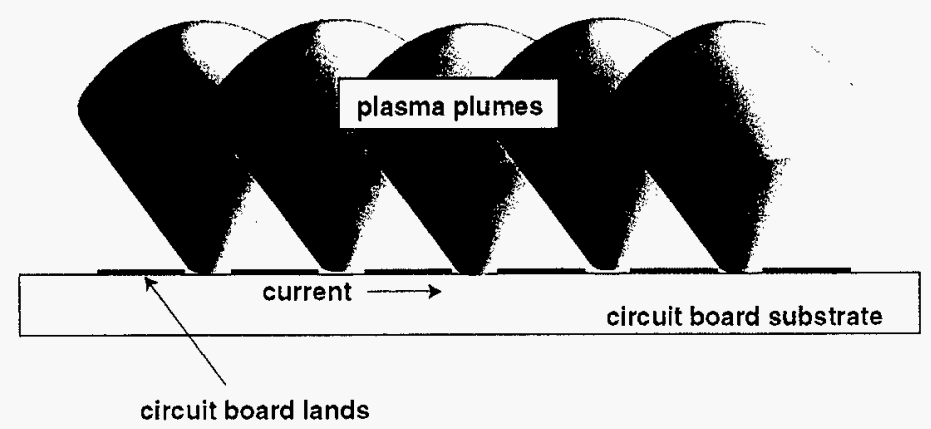

Figure 3-3. A DM-2 flashboard (left) and a conceptual drawing of plasma expansion from a board. There are 72 such flashboards (360 sparks) in the MCPOS. The drawing shows plasma about $100 \mathrm{~ns}$ after flashboard current begins.

The DM-2 main switch plasma source used many (72) spark surface-discharge flashboards. Each flashboard 30 has 5 sparks in series and is driven by a $50 \mathrm{nF}$ very lowinductance capacitor and circuit. A graphite suspension in alcohol applied to the spark region makes the discharge more reliable, and less affected by pressure. Each of the five gaps is $.75 \mathrm{~mm}$; with the graphite coating or carbon and metal vapor deposition from machine shots, the board sparks at six to eight $\mathrm{kV}$. Flashboards so conditioned spark over at the same applied voltage in atmospheric air or $10^{-5} \mathrm{~Pa}$ vacuum. Enhancing the carbon composition over hydrogen also lowers the space-charge limited ion loss current for a given switch gap. The fast risetime of voltage applied to the boards ( $200 \mathrm{~ns}$ ) further increases repeatability, and assures that all plasma sources operate within a few ns. Such pulse-charged systems are more efficient because of the faster current rise time and higher current. 27,31-33 Because of the extremely low inductance vacuum feedthrough connection from the pulse-charged capacitors to the plasma sources (about $20 \mathrm{nH}$ ), the current quarter-period time in the flashboards is about $50 \mathrm{~ns}$. This fast risetime allows efficient plasma generation before plasma short-circuits the plasma-creating surface discharge. Because of the low inductance of the flashboard circuit, a peak current of $15 \mathrm{kA}$ flows in each of the 72 flashboards. The total current flowing in all flashboards is about 1 MA, giving a total plasma source impedance of 0.01 , one to two orders of magnitude lower than most other systems of comparable energy. ${ }^{7,34,35}$ The fast, low-impedance plasma source system is very efficient and creates few neutrals.

The applied magnetic field near the plasma sources is nominally 6 Tesla. The applied magnetic field pressure is about ten times higher than the plasma stagnation pressure at $10^{16}$ carbon ions per $\mathrm{cm}^{3}$, with $400 \mathrm{eV}$ directed energy. 32 The dominating applied magnetic field causes the plasma to follow field lines as soon as the individual 
plasma plumes connect to form a complete azimuthal current path. Until the individual plasma spokes connect to form a ring, the magnetic field cannot exert a force on the plasma. The plasma plumes from the sparks have approximately a 45 degree half-angle; the plumes connect about $1 \mathrm{~cm}$ from the flashboards. At this distance, the density has dropped to roughly $10^{16}$ electrons per $\mathrm{cm}^{3}$. It is important that the sparks be located much closer together than the distance from the applied field coil to the flux excluder. This is because the plasma is not tied to field lines until the plumes connect, and the plasma crosses field lines (which are partly radial near the plasma sources) for a distance comparable to the spark separation, assuming a 45 degree plume half-angle. On DM-2 the applied field coil to flux excluder distance was about $6 \mathrm{~cm}$; the spark separation was about $1 \mathrm{~cm}$. Because the plasma crosses some field lines, the high-energy conduction electrons (with energies of $\mathrm{keV}$ to $\mathrm{MeV}$ ), which also follow field lines, do not strike the flashboards. This protects the plasma source. The applied magnetic field topology was configured so that the scalar total field decreased monotonically from the flashboards to the transmission line gap along a field line to avoid a magnetic mirror which would inhibit plasma motion.

These plasma sources also have the advantage that the easily changed flashboards, which are built with simple circuit-board technology, control the radial location of the plasma in the opening switch anode-cathode gap. This is possible because the applied magnetic field guides the plasma into the anode-cathode gap and the magnetic field lines arc largely axial in the anode-cathode gap; different applied field lines are at different radial positions in the gap. The effect of different radial plasma positions was not explored on DM-2 because of the limited time. The flashboards used on DM-2 were designed using simple plasma source energetic calculations, and magneto-static models of the switch region. The flashboards were designed to be protected from energetic electrons. Meltresistant tantalum plates were located where calculations showed the electrons would strike the anode in the switch region, and functioned as designed. The understanding of this plasma source allowed the source to adequately perform its function without hardware iterations or plasma interferometry.

\section{Fast magnetic field coil}

The fast magnetic field coil is the single most important part of the MCPOS. The fast coil (so called because it is energized on a $50 \mathrm{~ns}$ time scale) creates a magnetic pressure that applies a radial force to the plasma, and opens the switch. Central to this system is accelerating the conducting plasma with a magnetic field. ${ }^{34,36-39}$ Moving a plasma with a magnetic field on a microsecond or faster time scale is a well-studied phenomenon, and is used in plasma guns, plasma radiation sources, and plasma opening switches routinely.

Simple calculations can show the relative importance of magnetic pressure and plasma erosion. Assume a coaxial switch with a uniform plasma fill in a switching region. The magnetic pushback velocity assuming specular reflection from the sheath is: 


$$
V_{\operatorname{mag}} \geq \frac{B}{\sqrt{2 \mu_{0} n_{i} m_{i}}}
$$

where $B$ is the magnetic field, $\mu_{0}$ is the permeability of space, $n_{i}$ is the number density of plasma ions, and $m_{i}$ is the mass of the ions. The actual sheath velocity depends upon whether the pushback is snowplow or specular reflection; the lower value being specular reflection. To calculate the erosion rate, it will be assumed that no electrons come from the cathode into the plasma; this is an optimistic assumption for erosion velocity in most highpower POS experiments. Assume that ions and electrons leave the plasma as current flows through it, and no additional plasma enters the switch region. The erosion velocity $v_{e}$ is bounded by (depending on whether electrons go directly across the gap):

$$
\sqrt{\frac{m_{e} q_{i}}{m_{i} e}} \frac{I_{\text {switch }}}{2 q_{i} n_{i} A_{\text {swirch }}} \leq v_{e} \leq \frac{I_{\text {switch }}}{2 q_{i} n_{i} A_{\text {switch }}}
$$

where $I_{\text {swich }}$ is the generator current, $q$ is the ion charge state, $n_{i}$ is the number density of plasma ions, and $A_{\text {switch }}$ is the electrode area in contact with the plasma. Current and magnetic field are related by:

$$
B=\frac{\mu_{0} I}{2 \pi r} \sqrt{1+p^{2}},
$$

where $I$ is the generator current (always greater than or equal to $I_{\text {switch }}$ ), and $r$ is the cathode radius. The factor $p$ is the ratio of axial magnetic field to azimuthal field due to the fast coil; $p=0$ is no fast coil.

The minimum ratio of the two velocities is simply:

$$
\alpha=\min \left(\frac{v_{\text {mag }}}{v_{e}}\right)=\frac{q e A_{\text {switch }} \sqrt{1+p^{2}}}{\pi r} \sqrt{\frac{\mu_{0} n_{i}}{2 m_{i}}} .
$$

For example, consider a POS without a fast field coil, a doubly-ionized carbon plasma with density of $10^{21} / \mathrm{m}^{3}$, a cathode radius of $0.07 \mathrm{~m}$ and switch area of $0.05 \mathrm{~m}^{2}$. The magnetic pushback velocity is 12.9 times higher than the erosion velocity. For the MCPOS with a fast coil of $p=2.1$, and carbon at $5 \cdot 10^{20} / \mathrm{m}^{3}$, cathode radius of $0.125 \mathrm{~m}$, and switch area of $0.12 \mathrm{~m}^{2}$, the magnetic pushback velocity is 28.5 times the erosion velocity. In either switch, magnetic pressure clearly dominates erosion. These calculations are the lowest possible estimate for the ratio of magnetic to electric effects. The MCPOS is designed to be controlled by magnetic fields to the extent possible, enhancing the natural switch operation.

Fortunately, plasma motion due to magnetic pressure is more energy efficient than ion erosion. Ions, which are a loss mechanism in plasma opening switches, are accelerated to full switch voltage (for example $2 \mathrm{MeV}$ for $\mathrm{C}^{2+}$ at $1 \mathrm{MV}$ switch potential) if simply eroded from the plasma. Ions swept up in a magnetically accelerated sheath acquire twice 
the sheath velocity $(250 \mathrm{keV}$ for carbon at $100 \mathrm{~cm} / \mu \mathrm{s}$, a sheath velocity which would fully open the DM-2 switch in $20 \mathrm{~ns}$ ). One quickly sees that for this example, ions passively eroded from a plasma dissipate 8 times more energy than energy due to fast sheath motion.

Either a fast coil or a small-diameter center conductor will raise the local magnetic field. However, there are important differences that makes fast coils more desirable: First, a fast coil such as the one used on DM-2 has a much larger surface area than a single small center conductor. Cathode area in the POS region is important because of electrode plasma raised by conduction. The DM- 2 coil raised the total magnetic field at the center conductor (cathode) by a factor of 3.1 at a large diameter. To get the same increased field by reducing the diameter of the cathode would require a cathode one-third the diameter, with an equally reduced area. The second advantage of a fast coil is the magnetic field is nearly constant with distance from the coil. In contrast, the magnetic field decreases strongly with radius in a simple high-impedance coaxial transmission line. The third advantage of a fast coil such as used on DM-2 is that the azimuthal magnetic field in the POS region may be controlled with an opening switch (the trigger switch) shunting the fast coil. The trigger switch allows a low total field during the POS conduction, then raising the field for opening. This allows reduced plasma fill and faster opening. Simply using a small diameter center conductor raises the magnetic field for a given current, but the plasma density must be increased to keep the switch closed. The required increase in plasma density at least partially offsets the advantage of higher magnetic field. The fourth advantage of a fast coil is that the axial magnetic field from a fast coil in conjunction with the axial field from a low-voltage applied-field coil forms a system which prevents plasma translation, has a well-defined geometry, and is efficient. A fast field coil and slow coil system allows purely radial plasma motion. Restricting plasma motion to purely radial decreases the energy lost to unnecessary plasma velocity.

Before building any MCPOS hardware, the separatrix position for different ratios of applied field to fast field in the switch region is calculated. The separatrix location is the location of the plasma boundary if the plasma mass were zero. In practice the plasma boundary will not be the same as the separatrix location due to plasma inertia; early in time inertia causes the vacuum gap to be less than the separatrix location, and late in time inertia causes the vacuum gap to be larger than the separatrix location. Figure 5-13 shows separatrix and plasma boundary location for a DM-2 test. This is basically the figure-ofmerit for a switch design. A higher fast coil inductance creates a higher axial field for a given current, and thus applies a higher pressure to the switch plasma. The fast coil inductance is not a free parameter because of two limitations: first, the fast coil inductance should not be so large as to appreciably affect the output pulse. This implies that the fast coil free-space inductance should not exceed the product of the load impedance and the duration: $L_{\text {fastcoil }} \leq Z_{\text {load }}$ Pulsewidth. This is a conservative requirement because switch plasma excludes flux and lowers the fast coil inductance. As the switch opens, the inductance of the fast coil changes (this is a resistive term due to electrical energy transfer to plasma kinetic energy). The energy that goes into the fast coil to move plasma is lost to the load, but any opening switch has to move an armature, which requires energy. The second limitation on the fast coil is mechanical. For a given current, mechanical strain in the fast 
coil windings rises faster than fast coil inductance. Mechanical distortion of the fast coil can result in turn-to-turn shorting, which prevents proper MCPOS operation.

Through the middle of the fast field coil is a mechanical support rod. This rod has to support all the weight of the downstream transmission line and the cathode side of the load. This rod is also a current path that is electrically across the fast field coil. There are three major requirements on the size of this rod: First, the rod diameter must be small compared to the fast coil diameter so that fast field energy is in the MTTL gap, and not between the support rod and the fast coil. Second, the inductance of the rod must be much greater than the fast coil inductance. Third, the rod must mechanically support the weight of downstream hardware because the fast coil is not mechanically rigid. The first two requirements favor a small diameter support rod; the mechanical strength determines the minimum diameter. The DM-2 support rod was made as long as practical to maximize the inductance. The DM-2 support rod is $7.6 \mathrm{~cm}$ diameter and $76 \mathrm{~cm}$ long. The weight of the downstream hardware on DM-2 is about $50 \mathrm{~kg}$; the mechanical forces in the DM-2 support rod are well within material limits.

The fast coil and support rod circuits form an inductive current divider. Because the circuits are connected by metal, the flux is the same in the fast coil circuit and the support rod circuit. Measuring current in the support rod is a flux monitor because the support rod inductance is unchanging. Knowing fast coil current and flux, the time-dependent fast coil inductance can be calculated. On DM-2, the rod was 5.5 times more inductive than the free-space fast coil value. The peak current in the support rod was 100 to $150 \mathrm{kA}$ on most tests.

\section{Switch-load transmission line}

A 1.8 meter long, $5.5 \Omega$ transmission line carries power from the MCPOS to the load. An available $4.5 \Omega$ line was not used routinely because metal debris fragments from the load collected in the bottom of the transmission line, disrupting the power flow. This also happened with the $5.5 \Omega$ line, but the larger (more inductive) gap reduced the problem. The debris problem could be solved with an improved MITL mechanical design, or improved inter-shot cleaning techniques, or both. It is important to note that neither the 5.5 $\Omega$ nor $4.5 \Omega$ transmission lines would have worked if significant plasma translated out of the switch region. While all plasma opening switch systems have an axial force due to current through the plasma, the MCPOS has a mechanism (the applied field) which can oppose this force. Data from DM-2 and Tesla clearly show the absence of plasma translated downstream of the MCPOS. In contrast to the MCPOS, all inertial opening switch systems allow plasma translation downstream, affecting loads, transmission lines, and convolutes. Figure 3-4 shows the switch, transmission line, and load. 


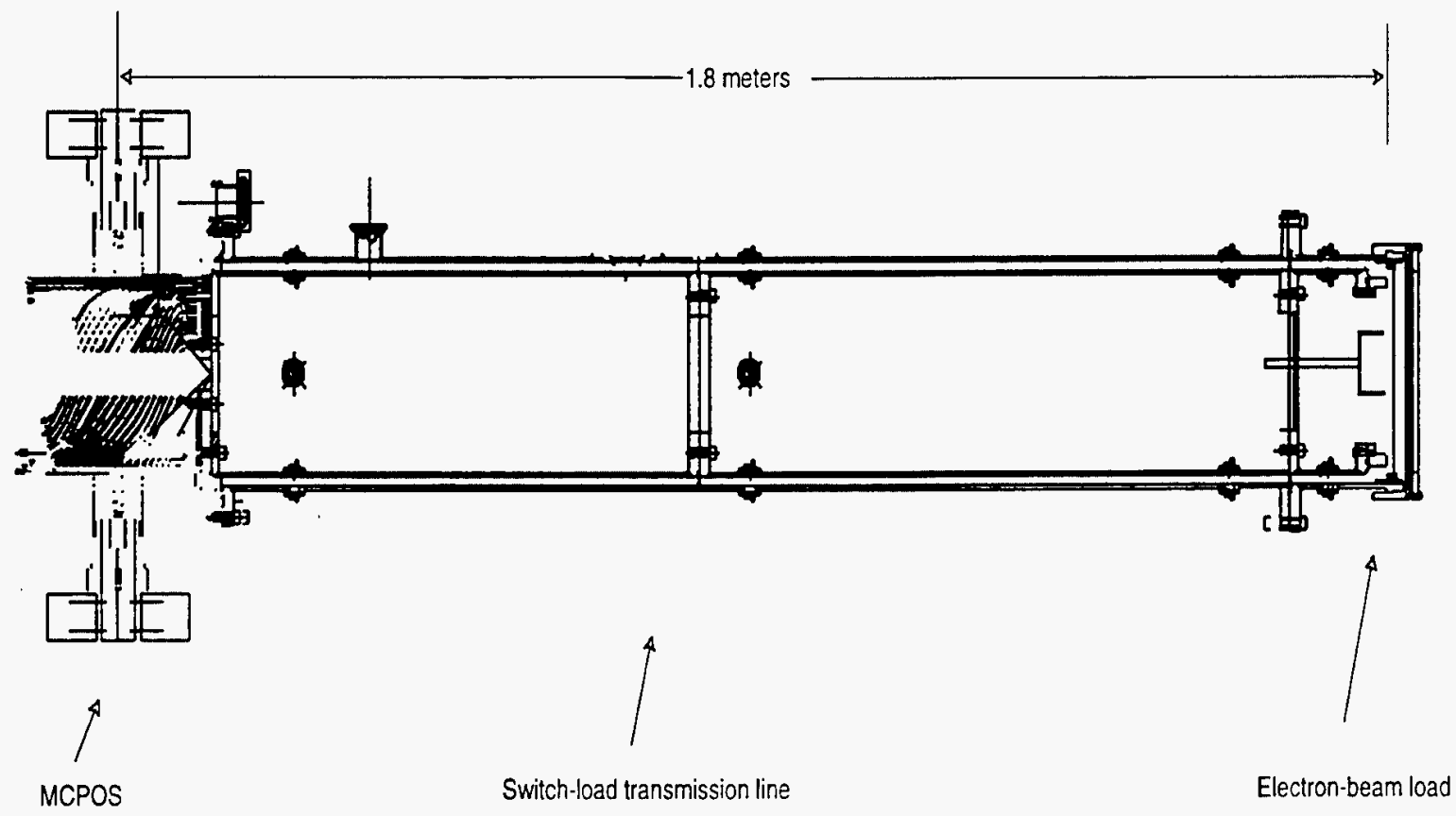

Figure 3-4. The switch, transmission line, and load on DM-2. The vacuum impedance of the transmission line is $5.5 \Omega$. The center conductor diameter is $24.2 \mathrm{~cm}$.

\section{Electron beam load}

The load used on DM-2 was at first a simple ring cathode with a planar anode. The poor impedance behavior (rapid impedance collapse) was improved by adding a small bias cathode. The $22 \mathrm{~cm}$ diameter cathode operated with $1 \mathrm{~cm}$ gaps typically. The bias cathode was $5.1 \mathrm{~cm}$ diameter and with gap equal to the main cathode drew one-fourth of the total current. The lack of translated plasma in the load region allowed significant flexibility in load parameters. Figure 3-5 shows the DM-2 load with the bias cathode. 


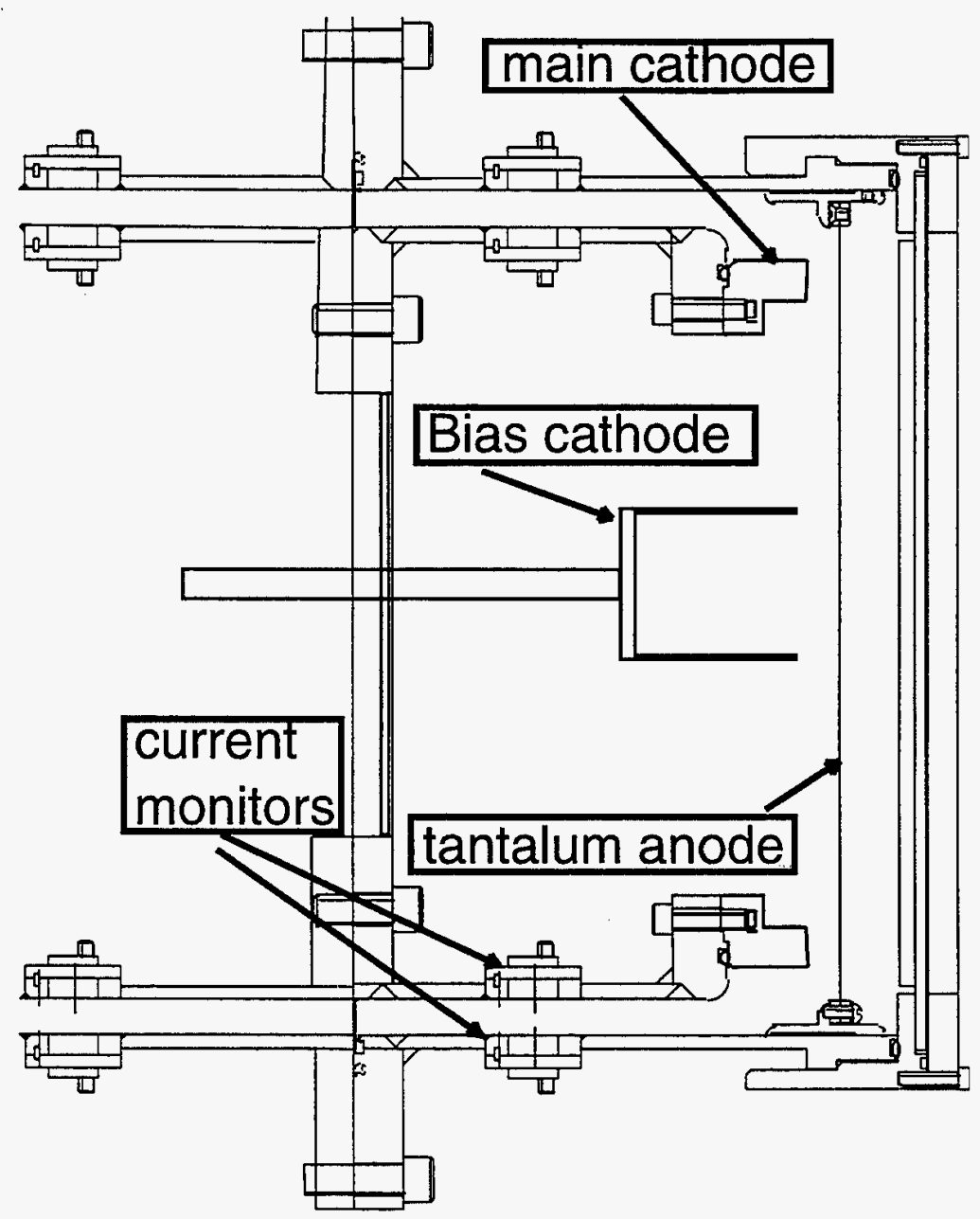

Figure 3-5. The load region of DM-2. The material to the right of the tantalum anode is the beam stop and vacuum window. This also shows the bias cathode, which was added near the beginning of the test series. 


\section{The DM-2 experiment and diagnostics}

\section{Chronology}

The MCPOS design for DM-2 began in September 1995. By January 1996 the design was complete except for a few mechanical details.

By late March, 1996 most of the parts were built. The plan was to test the switch on the Tesla pulser at lower current to identify any design problems, and proceed more rapidly on DM-2. Unfortunately, the funding was erratic, the project was completely unfunded for more than a month, and all team members had other full time commitments for the duration of the design and experiments. Because of these constraints, it was exceedingly difficult to get the 17 Tesla shots discussed below. However, those tests uncovered a vacuum power flow problem which was verified by Two-Quick particle-in-cell (PIC) modeling: $\mathrm{Br}$ field lines at the ends of the fast coil crossing the MITL gap caused an appreciable electron loss. 40 Further PIC modeling showed a simple solution which was implemented on DM-2 (moving the switch anode rods to larger diameter eliminated any significant loss).

The Tesla tests verified that the high-efficiency plasma sources were reliable. These sources met the MCPOS requirements for DM-2: a distributed source comprised of a large number of plasma sources (a total of about 500 individual sparks), high efficiency (leading to better reproducibility, less damage, a more highly ionized plasma, and fewer neutrals), high reliability (due to the low stored energy and the low voltage- $10 \mathrm{kV}$ peak), and low jitter ( $20 \mathrm{~ns}$ plasma source jitter with no prefires or misfires in over 1000 total firings).

The applied field coils were a concern because of the high magnetic pressures and the large diameter of the coils and flux excluders on DM-2. The short lead time precluded certifying coil vendors, so the coils were built in-house using techniques from the Inertial Confinement Fusion Ion Diode Program. The coils used titanium forms (somewhat more expensive than stainless steel, but much stronger and higher resistivity, which raises efficiency) and special magnet wire, vacuum-cast with a high-strength, shock resistant epoxy. Kevlar filament was wrapped over the coils in epoxy under tension, then a metal retainer ring was applied at high tension over the Kevlar. The result was a strong coil that was reliable on DM-2. In fact, while spare coils were built, only the original coil was ever used.

An applied field system design concern was the force on the vacuum chamber. Other programs (ion diodes, reflex triodes) using large applied fields have in the past had trouble with vacuum leaks induced by these forces. This problem was solved by placing the flux excluder in air, not affixed to the chamber. With the flux excluder coaxial around the applied field coil, there is no force other than hoop stress on the flux excluder itself. There were no problems with chamber leaks due to the applied magnetic field at any time during the DM-2 experiments. 


\section{Installation}

The experimental hardware and personnel arrived at Physics International, the site of the Decade module DM-2, on April 16th. Several improvements to the DM-2 facility were required to begin experiments. First, the MCPOS requires $10^{-3} \mathrm{~Pa}$ base vacuum for operation, which was beyond the DM-2 system capability. An additional pumping system solved this problem. In addition, the DM-2 signal cabling was too noisy for accurate data, so a remote data acquisition shielded box with digitizers, signal cables, and a data acquisition computer was installed for DM-2. Further complicating the experiments was the fact the DM-2 diode pointed at a close wall, requiring the long MITL to be disassembled (requiring 2 days) at every maintenance cycle. The hardware was designed to be disassembled as it would be on Decade, by removing the anode hardware in one piece. The experiments with the improved vacuum system and the portable Data Acquisition System (DAS) on DM-2 began on June 16, 1996.

The testing quickly showed that the MCPOS worked exactly as expected. However, the trigger POS, which energizes the fast coil of the main switch, worked more poorly than expected. Since the DM-2 MCPOS used an inertial POS for the trigger switch, there was essentially no way to predict the trigger switch performance, nor any way to scale the Tesla trigger POS to the DM-2 current levels. This is a common problem with scaling inertial plasma opening. The trigger switch needs to transfer two-thirds of the peak driver current into the fast coil within $50 \mathrm{~ns}$; on DM-2 the trigger switch never worked that well.

The experiments therefore concentrated on minor modifications to the trigger switch and making the best of the poor trigger switch performance. The schedule precluded any significant hardware modifications to the trigger switch. The schedule allowed about one month of optimizing and two weeks of radiation tests. The DM-2 Marx generators were more reliable at $65 \mathrm{kV}$ charge than at $85 \mathrm{kV}$ (the design point) so many tests were conducted at $65 \mathrm{kV}$ charge. The energy and power available from DM-2 at $65 \mathrm{kV}$ charge is about 60 percent of that available at $85 \mathrm{kV}$ charge.

The one month of testing at 65 and $75 \mathrm{kV}$ used stainless steel load anodes, which could tolerate multiple shots, and allowed several experiments without opening the vacuum chamber. The plasma fill time in the main switch, plasma fill time in the trigger switch, and the current in the applied field coil were varied, verifying that the MCPOS worked as designed.

The power transport in the MITL downstream of the MCPOS behaved as calculations predicted. Since there is no plasma translation downstream of the MCPOS, analytic and numerical models describe the power flow downstream of the MCPOS. In contrast, experiments using short sections of low impedance transmission line downstream of inertial plasma opening switches show poor power transport due to plasma translation into the MITL. The good power transport downstream of the MCPOS, and large cathodeplasma gap, proves it can drive loads through transmission lines, including systems where multiple modules are added in parallel. 
An important component in the MCPOS main switch was found to be deformed by the magnetic pressure upon it. This part, the Plasma Contact Electrode, surrounds the fast coil and carries current while the trigger plasma opening switch is closed. The deformation of this part eventually resulted in short-circuiting of the fast magnetic field coil. With the fast magnetic field coil shorted, the MCPOS operated about as well as a conventional plasma opening switch- far from adequate for the demanding task of driving power through the long downstream MITL. This problem appeared occasionally fairly early in the experiments, and worsened throughout the series. A solution was not devised and implemented until the last few days of testing. The solution prevented fast field coil shorting, but the small number of shots available at that point precluded a thorough evaluation.

\section{Diagnostics}

Several measurements must be made to characterize an opening switch system such as the MCPOS. These crucial measurements are voltage at the switch and at the load, and current (anode and cathode) upstream and downstream of the switch, and at the load. Both current and voltage were measured electrically, and not inferred. A plasma opening switch is an impedance (in fact, flow impedance ${ }^{41}$ ) device. Characterizing an opening switch by voltage without specifying drive current or load impedance is not useful. Likewise, it is impossible to understand the physics of a plasma opening switch with only current measurements, and worse yet with only anode current measurements. Axial electron flow, which is critical to these systems, can only be measured with both anode and cathode current monitors. For these reasons, which are common to all vacuum opening switch experiments, no test on DM-2 was performed without all of these electrical diagnostics.

On every experiment 35 current and voltage monitors (in addition to DM-2 machine diagnostics), were acquired on high-quality fast digitizers. The original DM-2 signal cables were replaced everywhere possible with high-quality shielded cables with high-quality connectors. On systems such as DM-2, essentially all signal noise voltage arises from the product of shield current and signal cable shield resistance. For that reason, it is important to use cable with a low resistance shield, and connectors with low resistance ground connections. BNC type connectors are notoriously poor in this respect. Type $\mathrm{N}$ connectors perform well, and SMA connectors are usually adequate. It would have been desirable to replace all $\mathrm{BNC}$ connectors, and that was done wherever possible. It was impossible to change the cables in the transit-time isolator, so the cathode signals that come through that isolator were noisier than anode signals. Every monitor (except those monitors which were high-impedance detectors) used a $10 \mathrm{nF}$ high-pass filter to block applied field coil baseline offsets. Every derivative-responding current monitor used a passive $\mathrm{RC}$ integrator, with a 2.4 microsecond time constant. These signals were numerically corrected for the small amount of filter roll-off and integrator droop.

Physics International calibrated the interface voltage monitors and the current monitors. The interface voltage monitors were calibrated using the current monitors on a short-circuit downline shot. The design of the interface voltage monitors precluded a calibration independent of inductance calculations and current monitor calibrations. 
Interface capacitive voltage monitors that cannot be independently calibrated are less desirable than monitors that can be calibrated with a moderate voltage calibration pulser. The current monitors were calibrated using a current transformer as reference, with a $20 \mathrm{kV}$ cable pulser. With careful calibrations, one could expect about 10 percent accuracy for the current monitors, and perhaps 15 percent) for the voltage monitors.

There were some problems with current monitors in the low-impedance MITL between the switch and the load. This problem was due to the fact that the current monitors were designed with large active area for large signals, but these large B-dots disrupted magnetic insulation in the MITL because the B-dot hole size was comparable to the MITL vacuum gap. The reduced magnetic insulation caused electrons to strike the anode B-dot monitors (at the downstream end) which eroded the monitor's epoxy, and eventually caused monitor failure. This problem is easily solved, but there was not time to get a full set of smaller B-dots built. The DM-2 design package included a design for smaller-area B-dots, and a set of four was built. The smaller B-dots worked, but there was inadequate time to have a full set of 20 made.

An Electron Launching Voltage Monitor 42 was installed on DM-2 just upstream of the MCPOS. This device is designed to measure voltage in low-impedance MITLs. This monitor can be installed just upstream of an opening switch, and measure voltage without large transmission line voltage corrections. Unfortunately, the region of transmission line available for the Electron Launching monitor was downstream of a radial transition. This monitor functions on electron flow, and can be affected by unstable flow from upstream regions. Although the total current in electron flow is low at the Electron Launcher location, the upstream transition may launch enough electrons ${ }^{14}$ to affect the probe operation. Also, the limited space forced the launching wire to be aimed downstream, which is undesirable. ${ }^{42}$ The electron launching monitors generally operated well until the time at which the fast MCPOS opening caused a high voltage out of the monitor. There may have been some high-voltage breakdown in the monitor itself or the signal cabling.

For these reasons, all the voltage measurements come from the interface capacitive monitors. To extrapolate this measurement to other locations, interface current monitors and a transmission line model 42,43 were used to calculate the voltage at the opening switch. A separate monitor measures voltage across the fast magnetic field coil and this is subtracted from the POS voltage for voltage just downstream of the POS. A transmission line model using current monitors in the MITL downstream of the switch corrects this voltage to the load location.

With sub-nanosecond DAS sampling rates (500 picosecond), and the MITL electromagnetic wave transit times of 6 to 10 nanoseconds, the most accurate method for voltage calculations is as follows: use passive integrators on the derivative-responding current monitors, perform numerical droop correction, then use this current in the transmission line model. The properties of the transmission line must be known for this process, specifically the impedance and the transit time. These can be calculated, and there are checks that can be done. First, the POS voltage is low while the switch is closed (there is no axial translation of MCPOS plasma which would cause a $\frac{d \phi}{d t}$ voltage. Second, 
previous tests at Sandia showed voltage drops of about 1000-2000 volts during conduction. In this way, the model can be tested before the MCPOS opened on every experiment where there is plasma in the switch. Third, the calculation from the switch to the load can be verified by short-circuiting the load. These tests were done routinely during the course of DM-2 experiments.

Radiation diagnostics were fielded by Physics International. A "calibrated" PIN diode was used on some DM-2 tests, the time integral of which generally agreed with the dosimeters. This detector was located 1 meter from the load, and thus presumably avoided saturation effects. This PIN diode was the only radiation data available for every shot, and was lightly filtered and had no equilibrator. The effect of missing the equilibrator is to reduce the signal on the PIN diode due to the absence of radiation back-scattered into the active region. Ideally, a bare, equilibrated, collimated far-field PIN (or a near-field PCD) diode would have been recorded on every DM-2 shot, but this was not done. An equilibrated, bare PIN diode is an accurate way to measure ionization rate in silicon (if the dose rate does not exceed about $10^{10} \mathrm{rad} /$ second), which can be accurately scaled to dose rate. Integrating dose rate gives dose. 44,45

Using the current and load voltage for every shot, a simulated X-ray detector signal is computed from:

$$
\frac{d \text { Dose }}{d t} \propto I_{\text {load }} V_{\text {load }}^{\beta}
$$

where

$$
1.8 \leq \beta \leq 2.9
$$

This is a basic formula for thick-target $\mathrm{X}$-ray production in the range 0.1 to 2 $\mathrm{MeV} .46$ The data presented in this report uses the low end of the accepted range for the value of $\beta$ to be as generally conservative as possible, and to be conservative in comparing stainless-steel to tantalum anodes. The bremsstrahlung cross section scales as the square root of atomic number, but the widely-different thickness of the stainless steel and tantalum converters affected the amount of radiation from the two anode types. Comparing this calculation to the measured PIN signal verifies the current and voltage calibrations.

\section{Voltage measurements in magnetically insulated transmission lines.}

Consider a section of transmission line as shown below. 


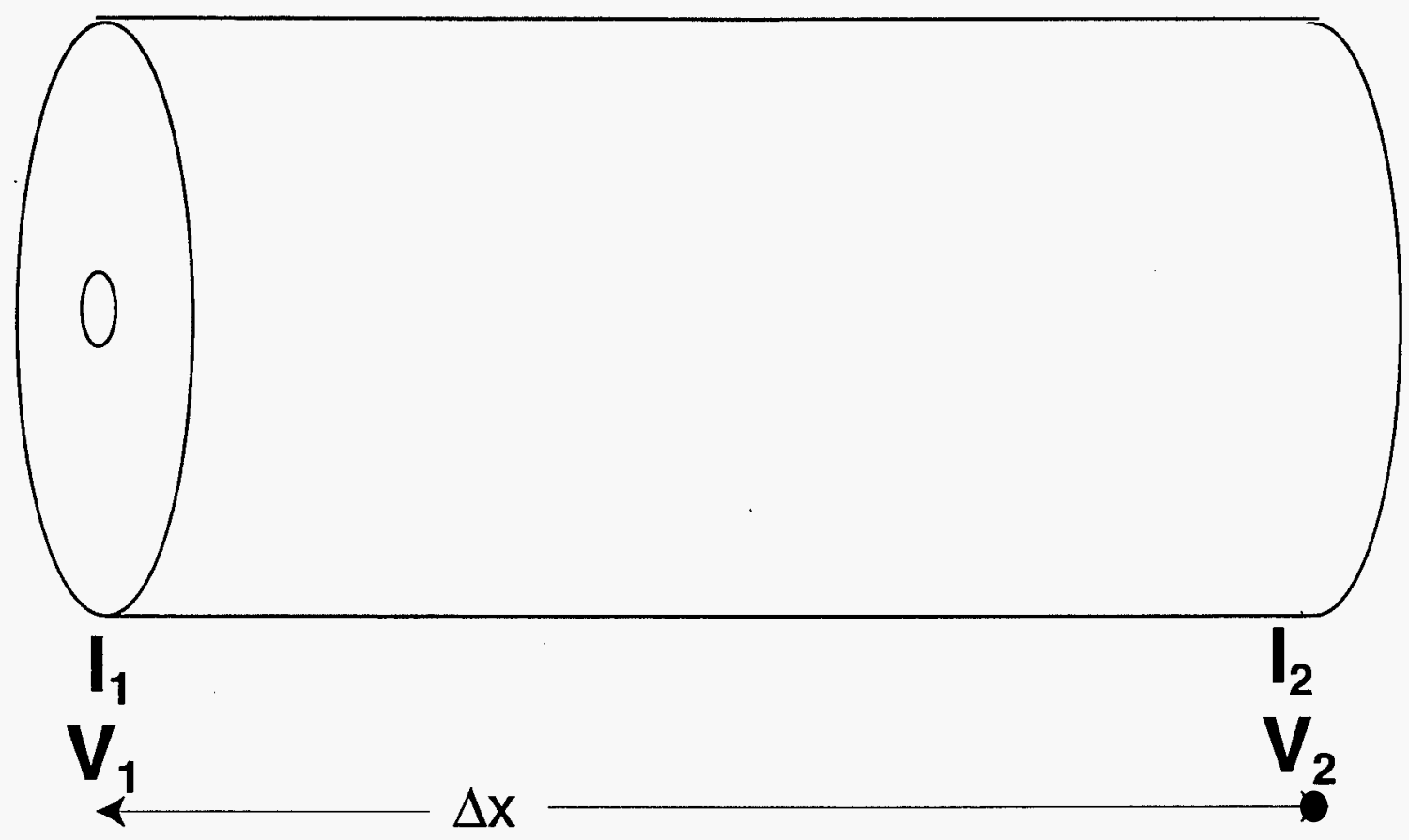

Figure 4-1. General transmission line model used for voltage extrapolation calculations.

This might represent for example, the transmission line between the vacuum interface and the magnetically controlled plasma opening switch. Power flows from left to right; the line has a length $\Delta x$, wave impedance $Z_{0}$, input voltage $V_{1}$, and input current $I_{1}$. The wave transit time for a vacuum transmission line is $\Delta t=\frac{\Delta x}{c}$, where $c$ is the speed of wave propagation in the transmission line dielectric. The desired quantity is the voltage at the right side of the transmission line, $V_{2} .42,43 \mathrm{In}$ an efficient system, like the MCPOS, it is known that the power flow is predominately axial. Measuring the current $I_{2}$ verifies this. Anode and cathode current are also measured. If the cathode current is a large fraction of anode current, then the vacuum wave impedance is a good approximation for the transmission line. If the flow impedance of the section is close to the vacuum impedance, then the vacuum impedance is a good approximation in that case as well. If there is a large vacuum-flowing electron current, then the impedance of the transmission line is effectively lower. In all the DM-2 MCPOS tests, the cathode current was a large fraction of the anode current (and between the switch and the load the vacuum impedance is the worst case for voltage drop). In such a system, an exact expression for the voltage $V_{2}$ is derived as follows:

The forward-going wave at location 1 is 


$$
V_{\text {forvard }}=\frac{V_{1}(t)+Z_{0} I_{1}(t)}{2}
$$

the reverse-going wave at location 1 is

$$
V_{\text {reverse }}=\frac{V_{1}(t)-Z_{0} I_{1}(t)}{2}
$$

With axial power flow, the voltage at location 2 is found by shifting the forward and reverse waves to location 2 , and add them to get the voltage:

$$
\begin{aligned}
V_{2} & =V_{\text {forward }}(t+\Delta t)+V_{\text {reverse }}(t-\Delta t) \\
& =\frac{V_{1}(t+\Delta t)+Z_{0} I_{1}(t+\Delta t)}{2}+\frac{V_{1}(t-\Delta t)-Z_{0} I_{1}(t-\Delta t)}{2} \\
& =\frac{V_{1}(t+\Delta t)+V_{1}(t-\Delta t)}{2}+\frac{Z_{0}}{2}\left(I_{1}(t+\Delta t)-I_{1}(t-\Delta t)\right) .
\end{aligned}
$$

The reverse-going wave demands a negative time shift to shift right; the forward-going wave shifts right for positive time shifts. For many situations, the voltage shifted term is close to the voltage at location 1 , so the approximation below is often used:

$$
V_{2} \cong V_{1}(t)+\frac{Z_{0}}{2}\left(I_{1}(t+\Delta t)-I_{1}(t-\Delta t)\right)
$$

The current shifts are important, however. The physical reason that the transmission line correction is more accurate than a simple derivative correction is as follows: the current $I_{1}$ is the sum of the output current and displacement current due to the capacitance of the transmission line: $I_{1}=I+I_{\text {disp }}$. The displacement current is roughly proportional to the rate-of-change of voltage. Using the derivative of $I_{1}$ adds a term proportional to the second derivative of voltage:

$$
\begin{aligned}
I_{1} & =I+I_{d i s p l} \\
\frac{d I_{1}}{d t} & =\frac{d I}{d t}+\frac{d I d i s p l}{d t} \\
& \cong \frac{d I}{d t}+\frac{d}{d t}\left(C \frac{d V}{d t}\right)
\end{aligned}
$$

since $C \approx \frac{\Delta t}{Z_{0}}$,

$$
\frac{d I_{1}}{d t} \cong \frac{d I}{d t}+\frac{\Delta t}{Z_{0}} \frac{d^{2} V}{d t^{2}}
$$

It is the second derivative of voltage that makes the inductive correction "noisy" for rise times even ten times longer than the transit time of the transmission line, and more 
noisy with shorter rise times. Even without numerical differentiation, the inductive correction is wrong; numerical differentiation adds numerical noise to worsen the calculation. Using the current at location 2 avoids the displacement current term, but miscalculates the flux in the transmission line.

Unless the rise times are well over ten times longer than the transmission line transit time, there is considerable error in using a simple inductive correction. The integral of voltage from an inductive correction will be correct, but the inductive correction oscillates about the correct solution. This can affect the peak voltage measurement.

\section{Current monitors in the DM-2 system.}

Because the current monitor output is proportional to the derivative of current, their signals must be integrated. Numerical integration masks digitizer noise, but can lead to large errors if the B-dot signal has high frequency components, as is often the case. Highquality passive integrators are more accurate if there are either signal features or noise with frequency components anywhere near the digitizer sampling rate. The integrators 47 used on DM-2 had a bandwidth of more than $500 \mathrm{MHz}$. Integrator response to a square-wave input is shown in Figure 4-2.

The integrators were tested by recording the output of the integrators with a $20 \mathrm{~V}$, 100-picosecond risetime input. The data were recorded with a $750 \mathrm{MHz}$ analog bandwidth, 10 picosecond/point digitizer and a $1 \mathrm{GHz}$ oscilloscope. The integrators had 2 millivolts (.001 percent of input) of spurious output which was damped in 2 ns. The time constant of the integrators was $Z_{0} C=2.4 \mu \mathrm{s}$. A $10 \mathrm{nF}$ high-pass filter before the integrator kept low-frequency applied field coil noise out of the integrator, which would manifest itself as a baseline offset. Since the applied field frequency was very much less than $\frac{1}{\tau_{\text {int }}}$, where $\tau_{\text {int }}$ is the integrator time constant, applied field baseline offsets would pass through the integrator without imposing a slope to the integrated signal. Before droop-correction of the signals, the average value of the first part of the waveform baseline was subtracted from the entire waveform. This prevented slope problems from the numerical integration of the signal. 


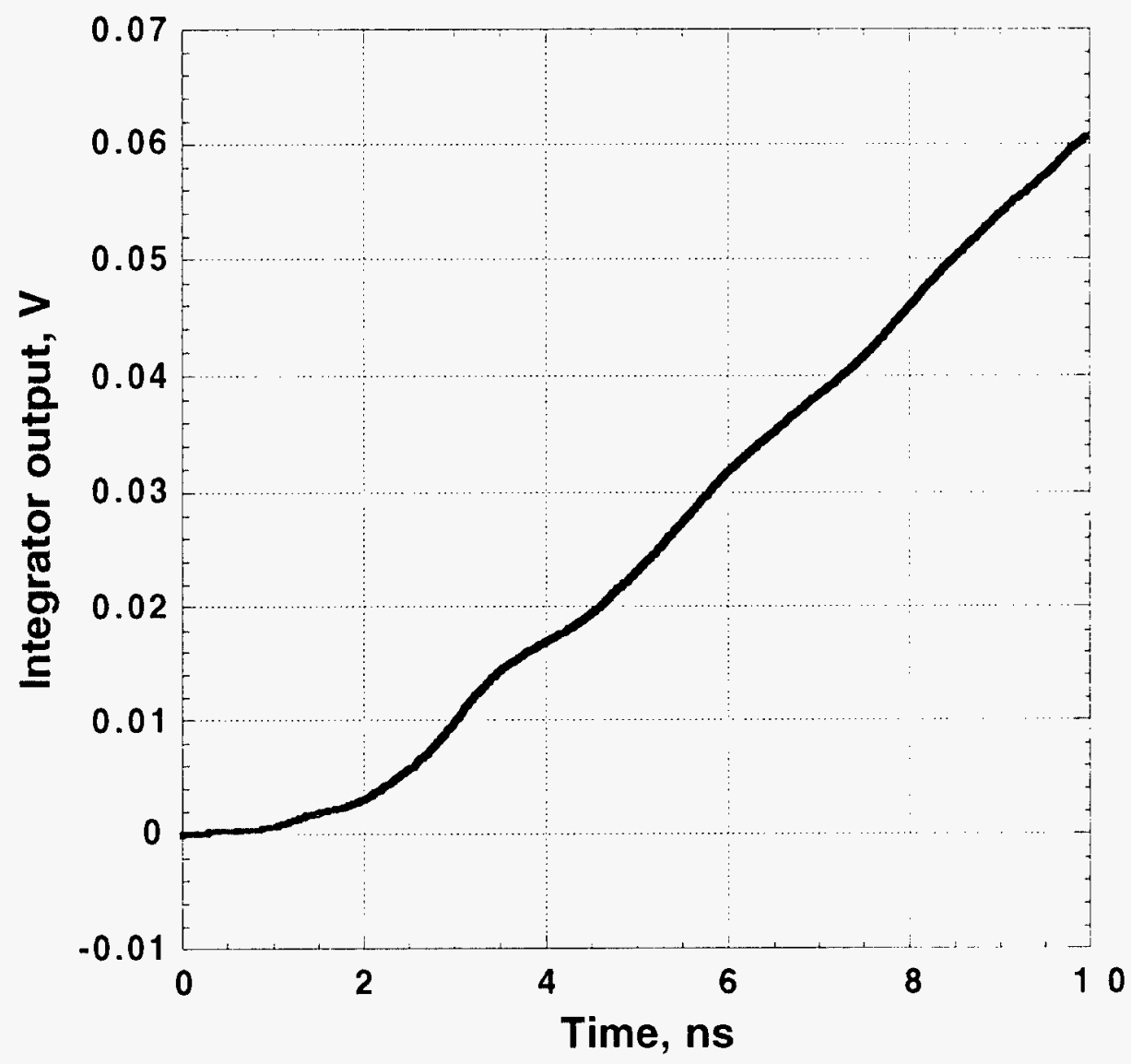

Figure 4-2. Output of the integrators used on DM-2 with a $20 \mathrm{~V}, 100$ picosecond rise time square wave input. A perfect integrator would give a linear ramp.

\section{Metrics of opening switch performance}

There is a large body of work devoted to studying terawatt power flow in vacuum. $11-16,23,41,48-50$ of the models available, flow impedance is used here to relate electrical measurements to physical conditions in the opening switch, as well as in the MITLs upstream and downstream of the opening switch. The flow impedance model (as applied here) can be thought of as using well-developed electron flow theory to relate 
vacuum gaps, voltage, and electron flow in a MITL. 41,51 The vacuum gaps may be between plasmas, or between plasma and metal, or between two metal conductors. In the case of (unmoving) metal conductors, the vacuum gap is known, and can solve equations for either voltage or electron flow. In the case of moving plasma boundaries, voltage and electron flow is measured, and from those an effective vacuum gap can be inferred.

The concept of flow impedance comes from experimental observations, theoretical models, and computer simulations of magnetically-insulated systems. Flow impedance in a MITL near a perturbation (e.g., an opening switch plasma) is

$$
Z_{f l o w} \equiv \frac{V}{\left(I_{u a}^{2}-I_{d c}^{2}\right)^{\frac{1}{2}}}
$$

where $Z_{\text {flow }}$ is the flow impedance of the region, $V$ is the voltage in the region of interest, $I_{u a}$ is anode current upstream of the perturbation, and $I_{d c}$ is cathode current downstream of the region of interest. The downstream cathode current monitor does not register vacuumflowing electrons launched in the increased electric field of the switch region. ${ }^{17}$ If there is no perturbation which increases electron flow (an opening switch plasma, or a convolute, or a power flow asymmetry 52,53 ), then the flow impedance of a transmission line will be close to, but less than the vacuum wave impedance if the voltage is such that electrons are relativistic (greater than $500 \mathrm{kV}$ ). Downstream of a perturbation, however, the flow impedance will remain close to the local geometric impedance of the perturbation, and not necessarily that of the local transmission line. This is because electrons cannot return to the cathode, and are driven downstream by the self-magnetic field. The flow impedance of an opening switch, for example, is related to the vacuum gap in the switch region. Because electrons cannot generally return to the cathode, the flow impedance downstream of a plasma opening switch remains close to the minimum flow impedance in the switch region. This is very important because it allows measuring the opening switch sheath width in the POS, with diagnostics outside the harsh environment of the POS. Flow impedance can be related to a physical gap. However, for circuit simulations and theoretical calculations, the flow impedance itself is the most informative parameter. The ratios of switch flow impedance to driver impedance, load impedance, and transmission line impedance give simple but important power-flow figures of merit for any opening switch system. 


\section{Results}

\section{Opening due to fast coil magnetic pressure}

The MCPOS relies upon plasma acceleration by force from a magnetic ficld. An experiment to measure the effect of the fast field was conducted early in the experimental run. A straightforward experiment shows the that the plasma is tied to applied magnetic field lines. Figure 5-1 shows upstream and downstream currents on an experiment with moderate plasma fill times and applied field magnitude.

DM-2 (normal applied B polarity)

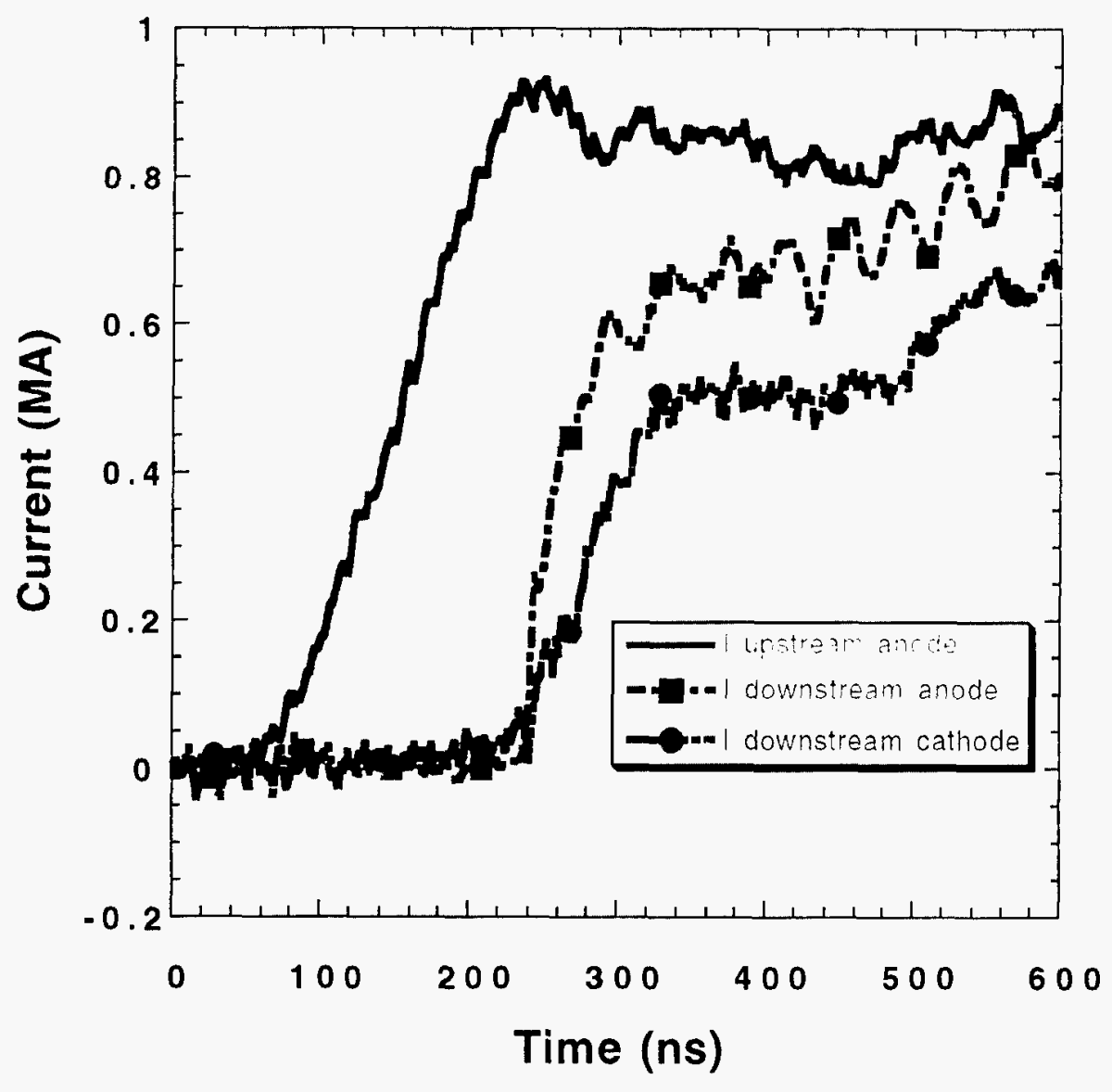

Figure 5-1. Current measurements on a DM-2 test with normal applied field polarity, i.e., the applied magnetic field is in the same axial direction as the fast magnetic field. This is the normal operating mode. 
Figure 5-2 shows upstream and downstream currents for an identical test, except with a reversed applied field. With the applied field reversed, there is no separatrix, and the switch does not open. These tests verify that the fast axial magnetic field (and not ion erosion or azimuthal field pressure) opens the MCPOS.

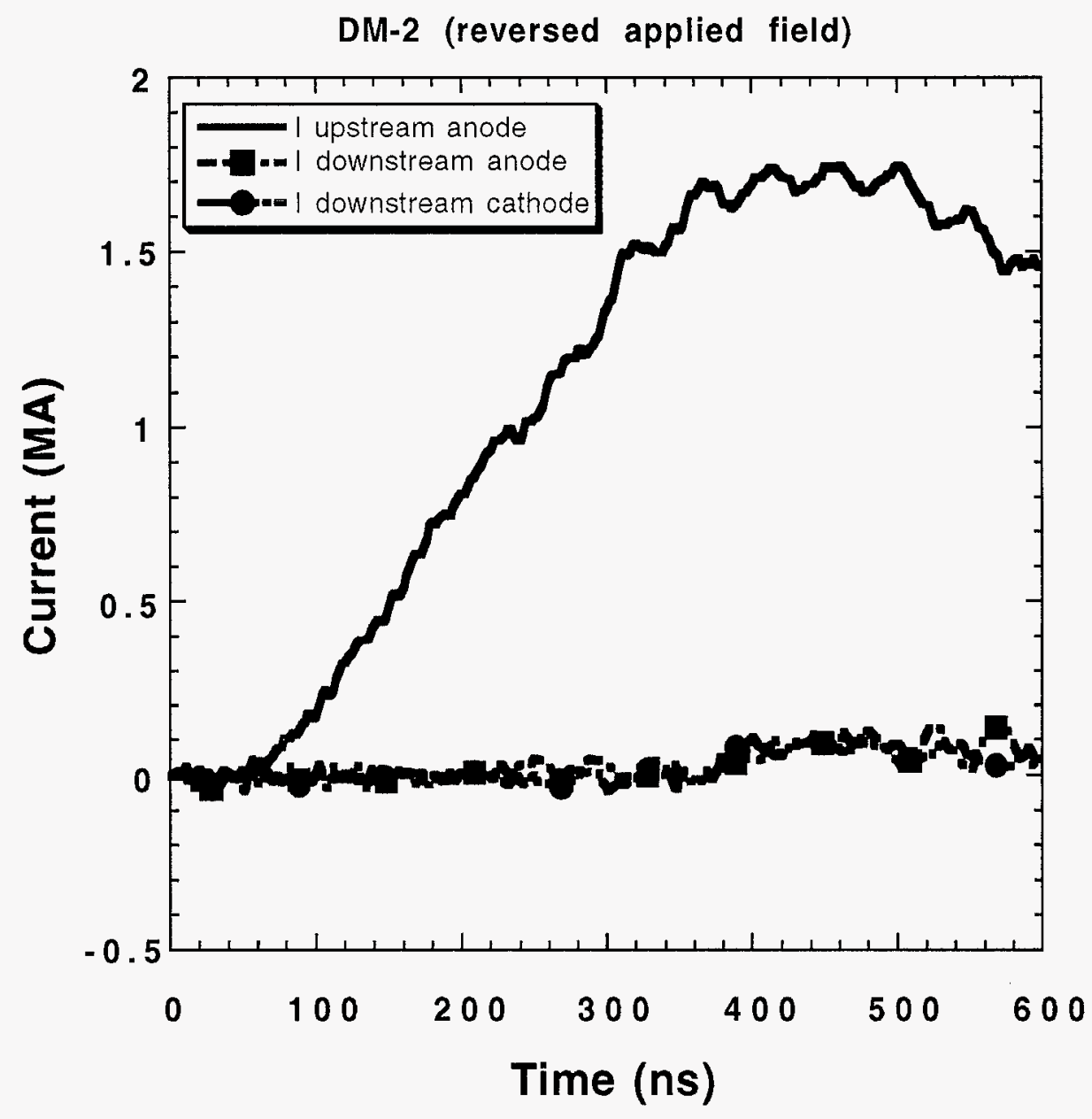

Figure 5-2. Current measurements on a DM-2 test with the applied field opposite the direction of the fast field. The MCPOS cannot open under these conditions because there is no radial pressure from the axial fast field. 


\section{Trigger switch and MCPOS performance}

As described before, there are two separate opening switches in the MCPOS system. The trigger switch, whose function is to delay the onset of current in the fast coil and to make the fast coil current rise faster than that of the driver, is just upstream of the main switch. The second switch, the MCPOS must short-circuit the MITL until nearly peak driver current, then open, and allow power to flow to the load. The load impedance will be higher than the driver impedance, so the load voltage should be higher than the driver voltage. The MCPOS and trigger switches are essentially in series, and the trigger switch must open first. The discussion will begin with the trigger switch.

The trigger switch on DM-2, as well as on Tesla, was a simple inertial POS. Since this switch drives the fast coil (which is on the cathode), this switch can be no larger than the cathode diameter in the switch region without a complicated power fecd and convolute system. The DM-2 experiments had the center conductor as the cathode; on Tesla the cathode was the outer conductor. The DM-2 experiments were higher current and smaller cathode diameter, so the linear current density in the trigger switch was a factor of 3.75 higher on DM-2. The greatly increased current density requires increased plasma density in the trigger switch region. Though there are many opinions as to the best way to design this type of inertial POS, there are compelling reasons for building opening switches in larger, rather than smaller volumes- for example, lower electrode energy deposition and reduced surface plasmas. The small trigger switch volume required high plasma density to keep the trigger switch closed, which leads to small opened gaps. Small opened gaps in a plasma opening switch can lead to increased effect of electrode surface conditions and often, poor opening and re-closure of the switch. The space available on DM-2 simply precluded building a larger trigger switch.

The trigger switch drives the fast coil, which starts at approximately zero inductance, and can rise to nearly the vacuum value of $28 \mathrm{nH}$. This dynamic inductance behaves like a resistor of value $Z=\frac{d L}{d t}$. If the main switch opens completely in $20 \mathrm{~ns}$, the trigger switch load is about $1.4 \Omega$. The trigger switch flow impedance must therefore be at least $1 \Omega$ to drive a large fraction of the machine current into the fast coil. Only cathode current downstream of the trigger switch will drive the fast coil; vacuum-flowing electrons are lost just upstream of the fast coil. The effect of these lost electrons could be seen as damage on the inner surface of the plasma contact electrode just upstream of the fast coil. On DM-2 however, the trigger switch performed more poorly than desired. Figure 5-3 shows the flow impedance of the trigger switch, and flow impedance of the MCPOS on a DM-2 experiment. The ratio of the MCPOS flow impedance to the trigger switch flow impedance is a measure of the impedance gain of the MCPOS. Note also that the relatively narrow temporal width of the trigger switch flow impedance peak indicates re-closure. This is typical of high plasma density, high current density inertial opening switches. The trigger switch flow impedance for the DM-2 experiments was in the range of 0.2-0.4 $\Omega$. The consequently reduced fast axial magnetic field did not push plasma out of the MITL gap as far as desired. 


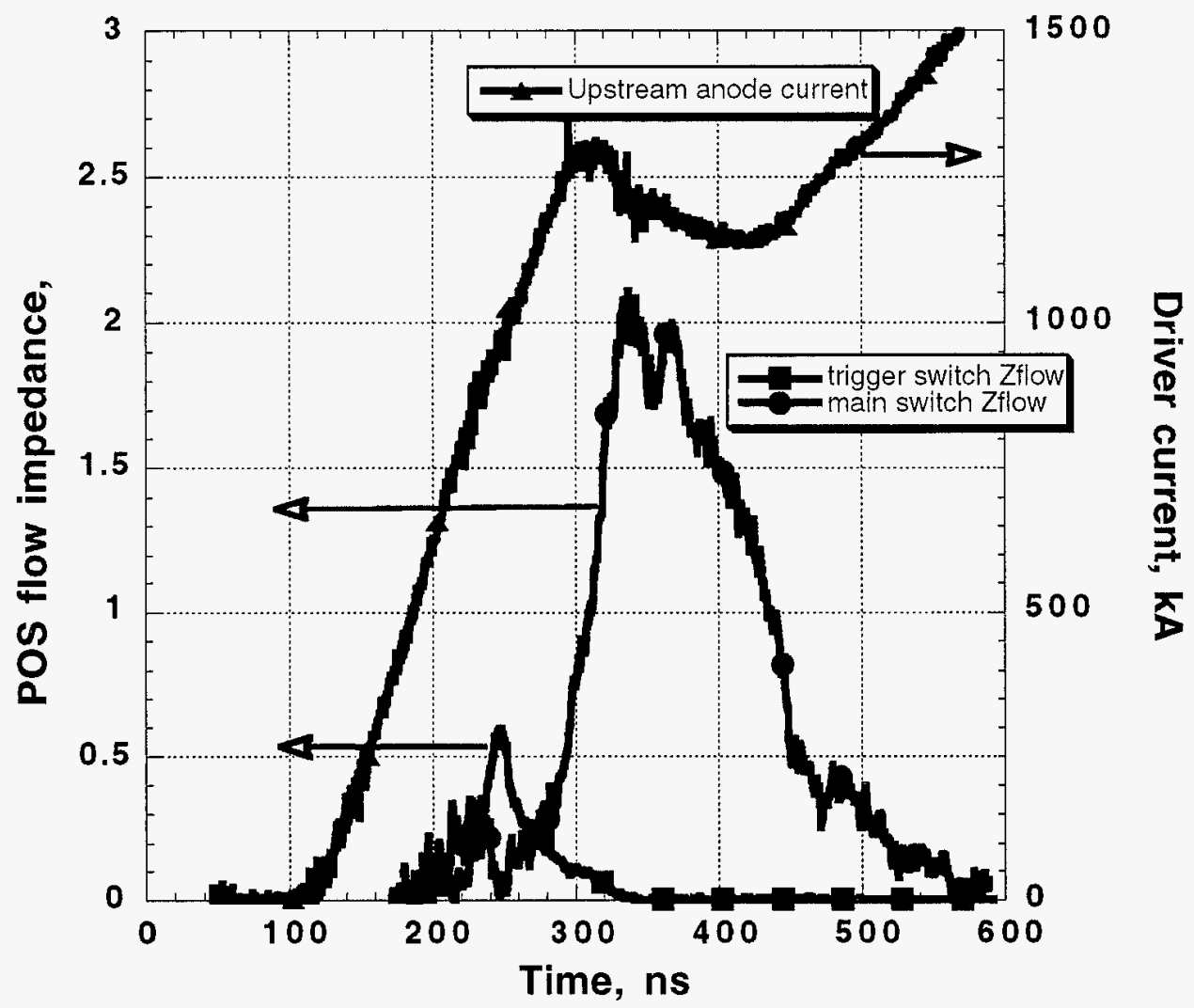

Figure 5-3. Trigger switch and main switch flow impedance as a function of time on DM-2 shot 339. Also shown is the driver current for timing information.

Figure 5-4 shows the effective transmission line vacuum gap in the switch region for the best DM-2 test, number 243. The effective gap is calculated from gap $=r_{\text {cathode }}\left[\exp \left(\frac{Z_{\text {fow }}}{60 \Omega}\right)-1\right]$, where $r_{\text {cathode }}$ is the cathode radius, and $Z_{\text {flow }}$ is the POS flow impedance. 


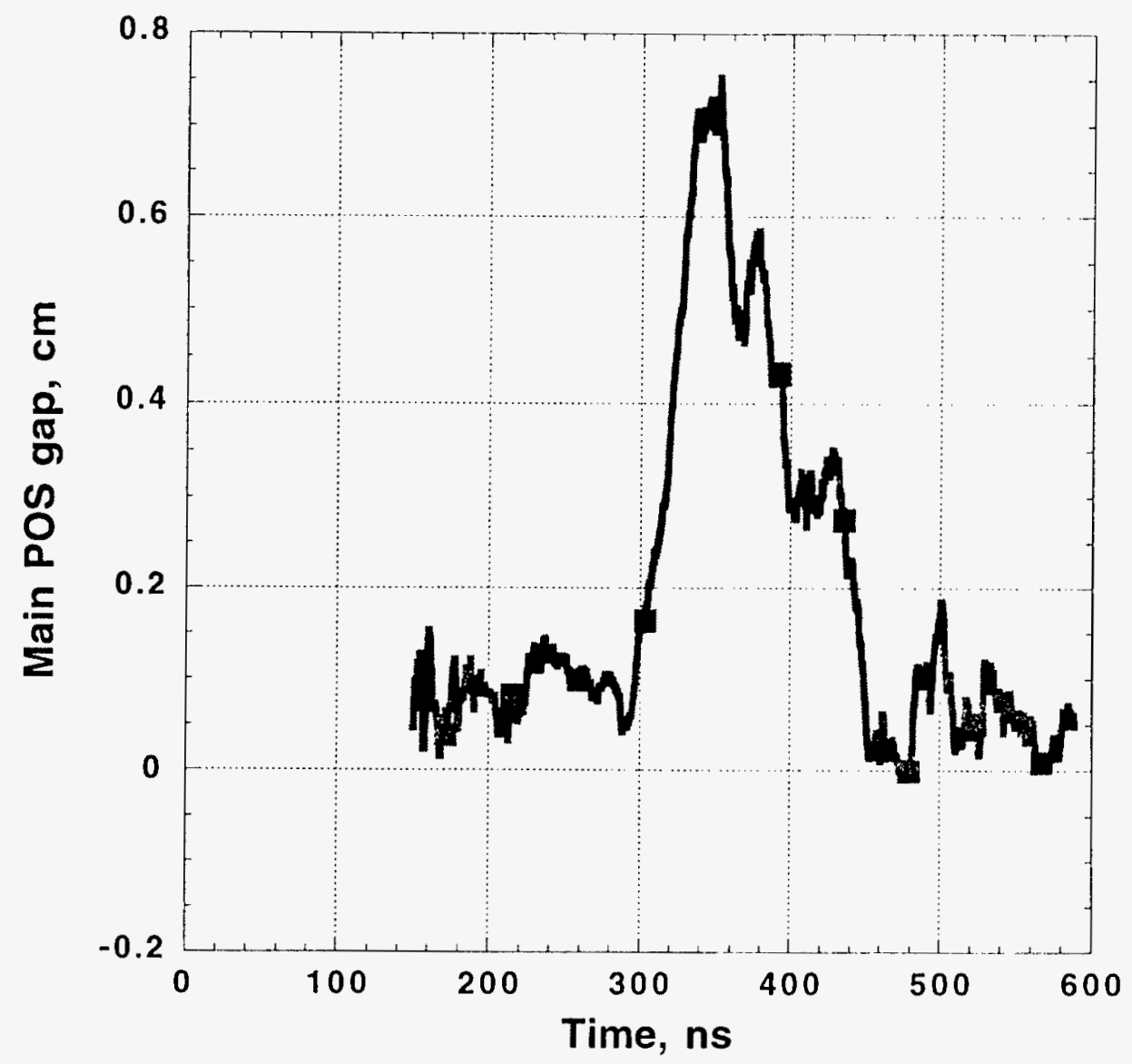

Figure 5-4. Calculated effective transmission line gap, on the best DM-2 experiment. This is a direct conversion from flow impedance to gap.

Figure 5-5 shows the current monitor data for this experiment. The high fraction of total current in the downstream cathode monitor shows that the load is determining switch voltage, and that power transport downstream of the switch is efficient. The currents shown in Figure 5-5 are upstream anode current, fast coil current, and anode and cathode currents downstream of the MCPOS on DM-2 shot 243. In spite of the poor trigger switch operation shown by the slow fast coil current risetime, the MCPOS operation is excellent. Load and switch are voltage determined by the load impedance, and are not limited by the opening switch. Current at the downstream end of the MITL is 100 percent of current at the upstream end of the MITL. 


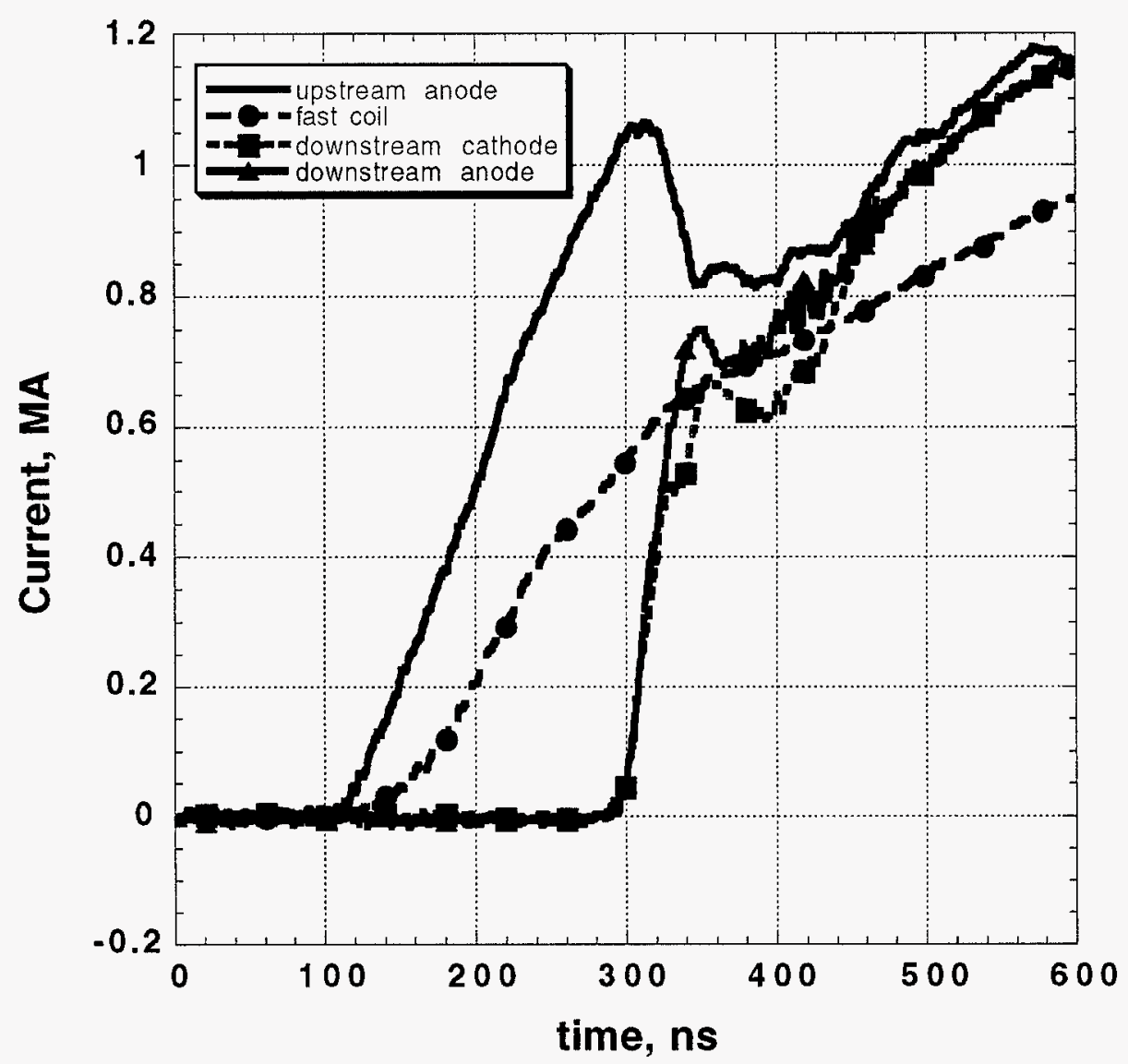

Figure 5-5. Currents on DM-2 for the best DM-2 experiment. The currents show the poor operation of the trigger switch, leading to the slow rise of fast coil current.

Figure 5-6 shows voltages calculated throughout the DM-2 system. The plasma opening switch is the highest voltage point in the system, as it should be. The load voltage is lower than the interface voltage due to the faster rise time of current downstream of the opening switch. The rise in interface voltage when the switch opens shows that the interface does not flash, and that the impedance upstream of the interface is comparable to the vacuum impedance. The relatively small difference between switch and interface voltage shows that the correction done to the interface waveform is relatively small. 


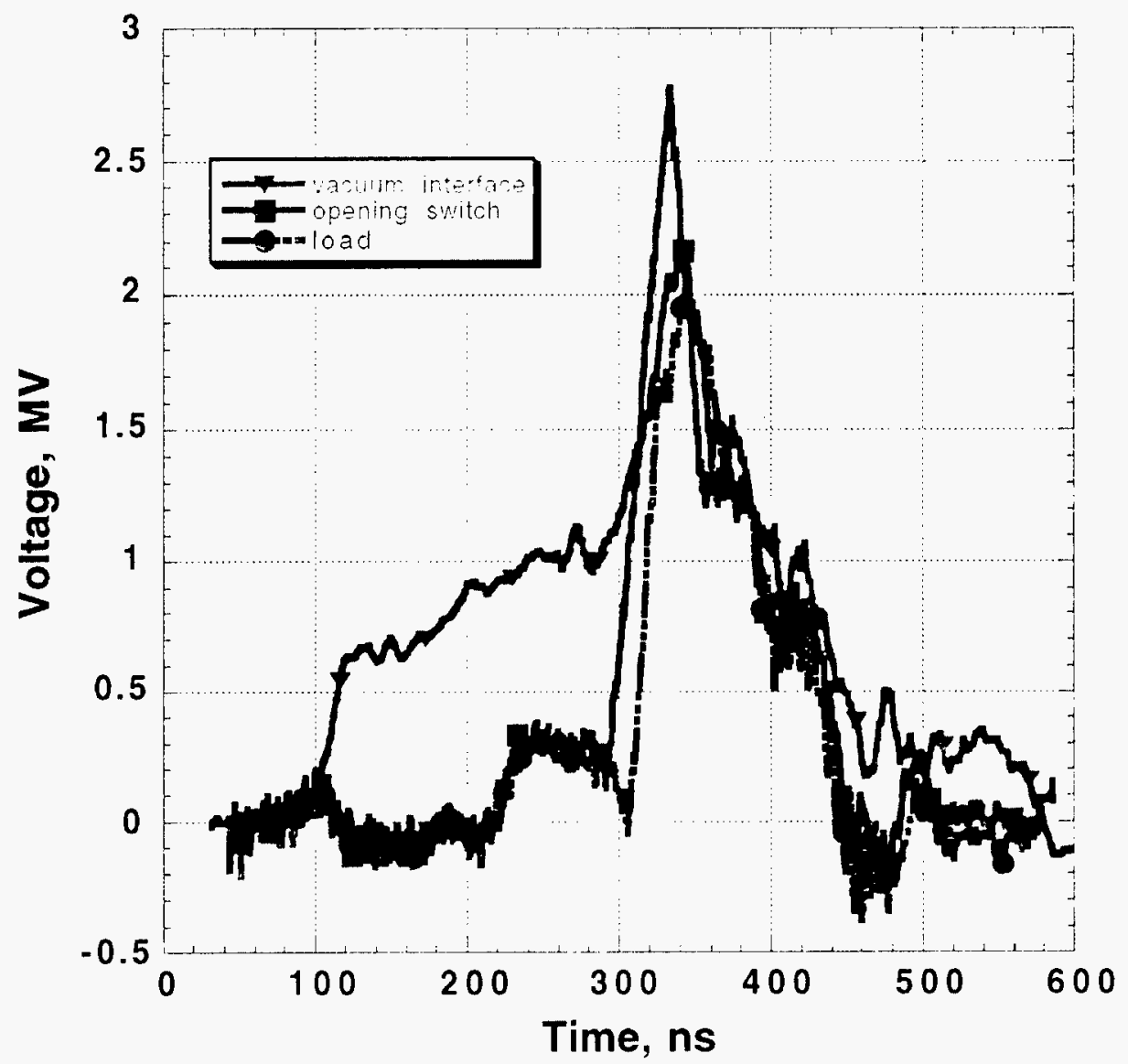

Figure 5-6. Calculated voltages throughout DM-2 using a full, exact transmission line equation for DM-2 experiment 243.

Figure 5-7 shows the DM-2 main switch opening current as a function of trigger switch opening current. The spread is due to variations in applied magnetic ficld and main switch plasma fill mass. The spread is worsened by the slow opening rate of the trigger switch. It is clear that the trigger switch opening time influences the main switch opening time. 


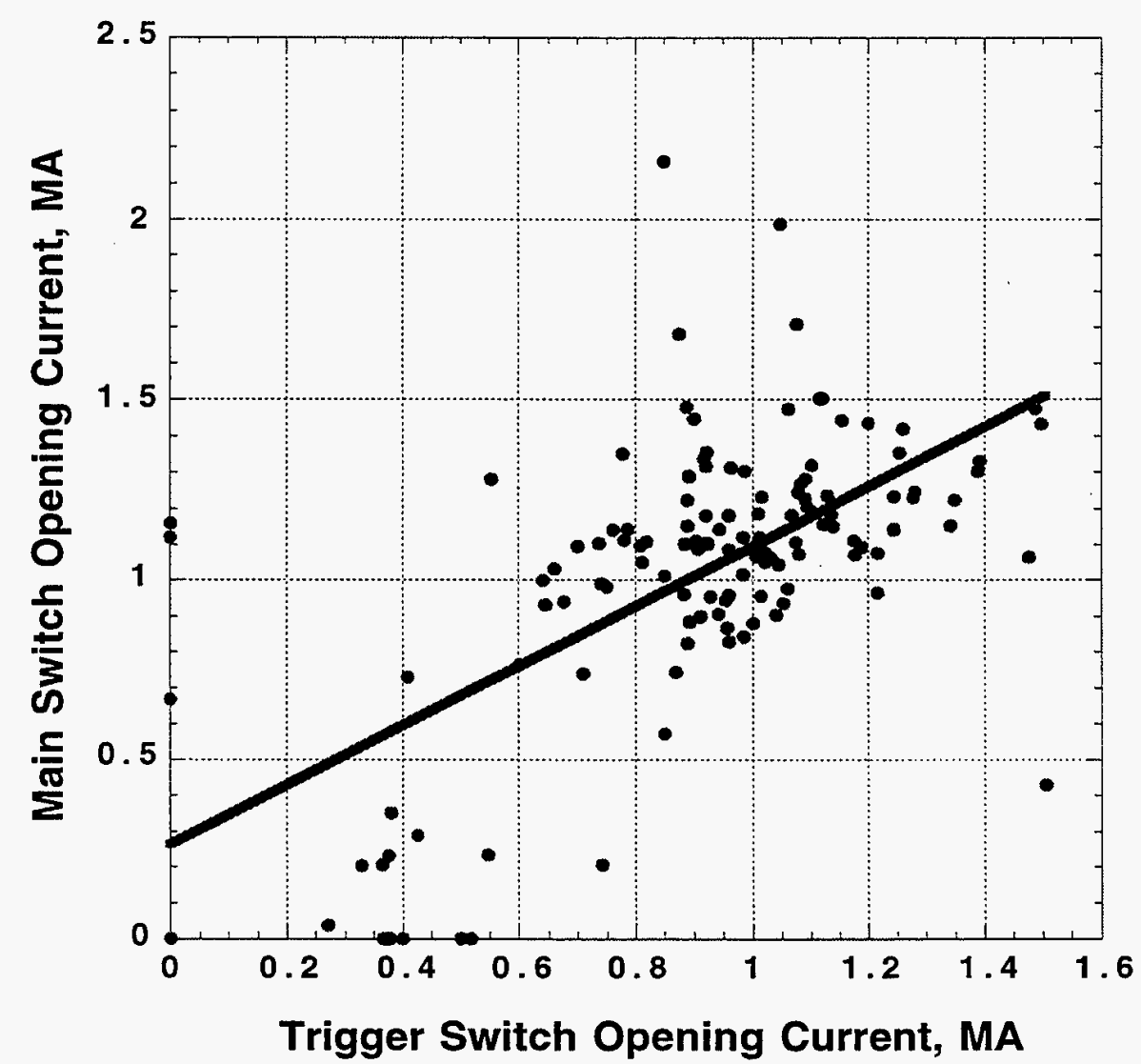

Figure 5-7. DM-2 main switch opening current as a function of trigger switch opening current. This shows all DM-2 tests. The non-zero yintercept is because of main switch plasma inertia. Zero values of opening current indicate no plasma in the respective switch.

Figure 5-8 shows the main switch opening current as a function of main switch plasma fill time. At zero fill time, the opening current is zero. The fast plasma sources on DM-2 make a plasma burst in a time short compared to typical fill times; plasma mass variations with fill time are due mostly to velocity spread in the plasma. 


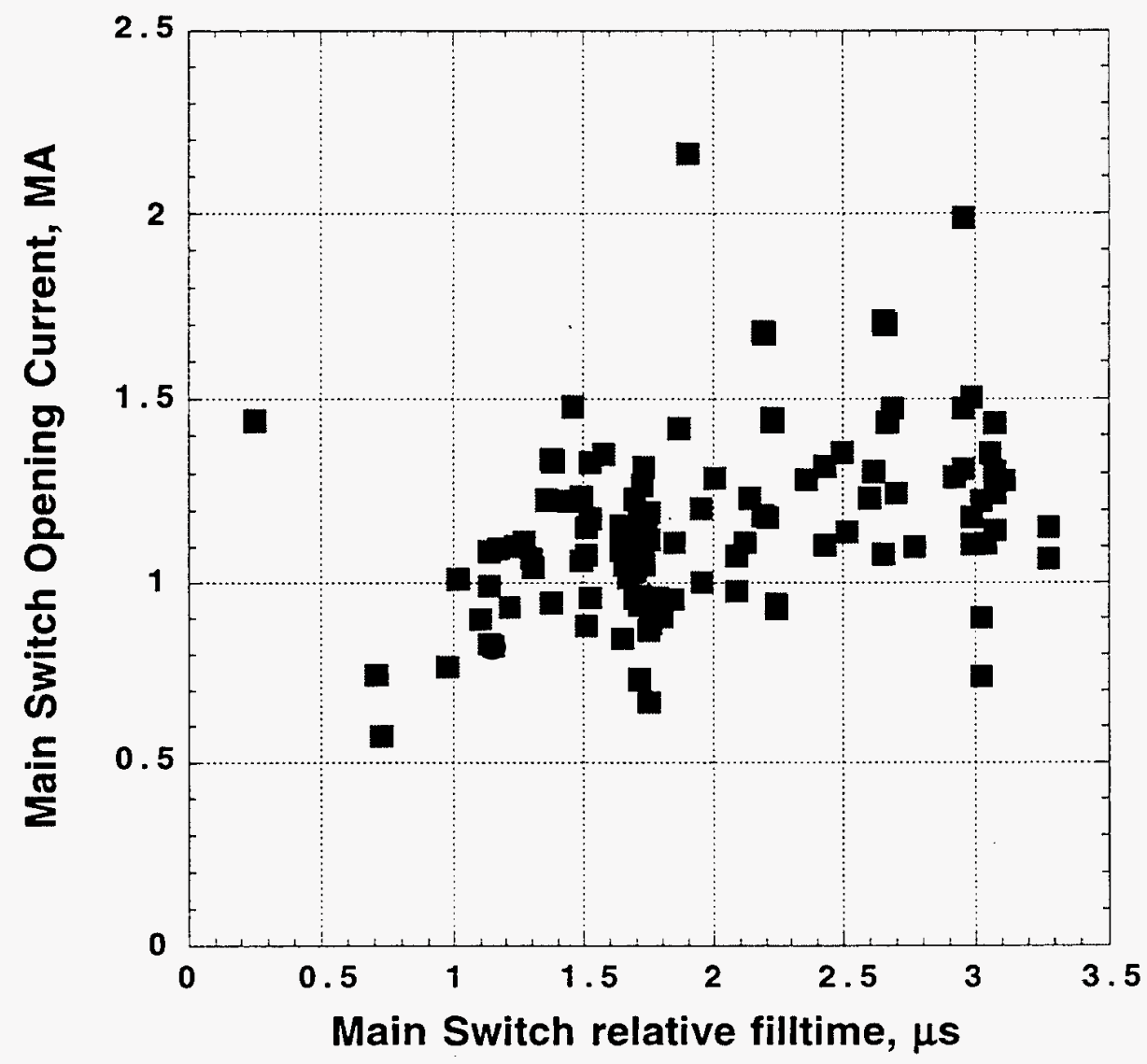

Figure 5-8. DM-2 main switch opening current versus the plasma fill time of the main switch. At zero plasma fill, the opening current will be zero, but in the operating range opening current is only slightly dependent upon plasma mass.

Figure 5-9 shows the performance of the DM-2 trigger switch versus trigger switch opening current. The falling performance of the trigger switch with conduction current was a problem on DM-2. The spread in the performance at a given conduction time is also evident. Both properties are undesirable for any opening switch system. The large variations in trigger switch performance at a given conduction current affect the performance of the MCPOS. 


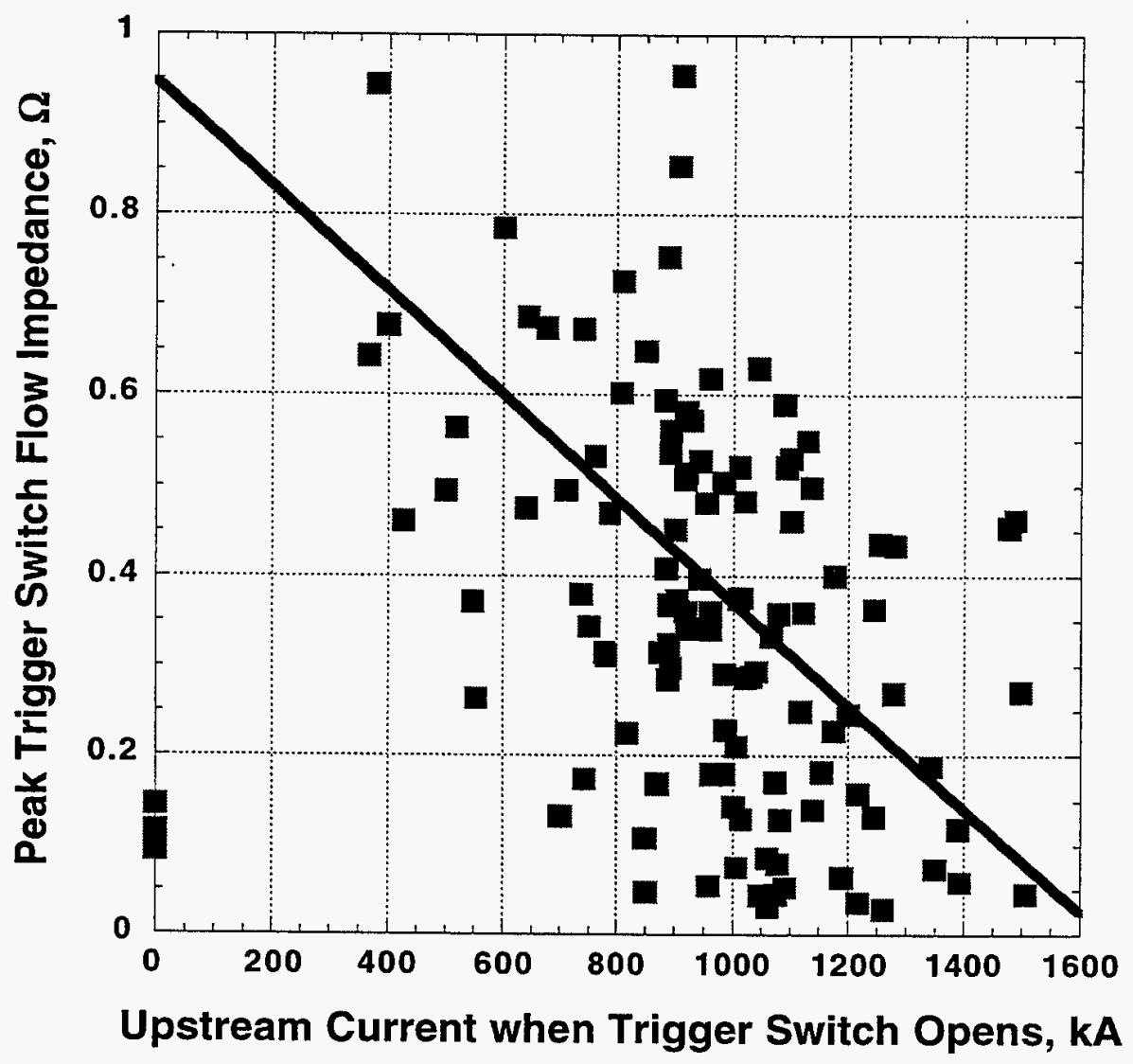

Figure 5-9. The trigger switch flow impedance (at peak main switch voltage), versus the trigger switch opening current switch for all DM-2 shots. The trigger switch performance rapidly falls with conduction current, and there is a large spread in trigger switch performance at a given opening point.

The MCPOS was limited by the trigger switch, and itself was not a limitation on DM-2. Figure 5-10 shows the main switch flow impedance versus the main switch opening current. The main switch flow impedance falls with higher conduction current, due to the decreasing performance of the trigger switch. Figure 5-11 shows the ratio of MCPOS flow impedance to trigger switch flow impedance, versus MCPOS opening current. This curve can go to high values, because the main switch will generally open even if the trigger switch does not open. The vertical spread is due to the varied MCPOS plasma fill times and magnetic field. Also shown on Figure 5-11 is the line indicating the design point of the DM-2 MCPOS, a ratio of three. This comes from a MCPOS flow 
impedance of $3 \Omega$ requiring a trigger switch flow impedance of $1 \Omega$ to drive adequate current in the fast coil. The MCPOS always exceeded the required performance.

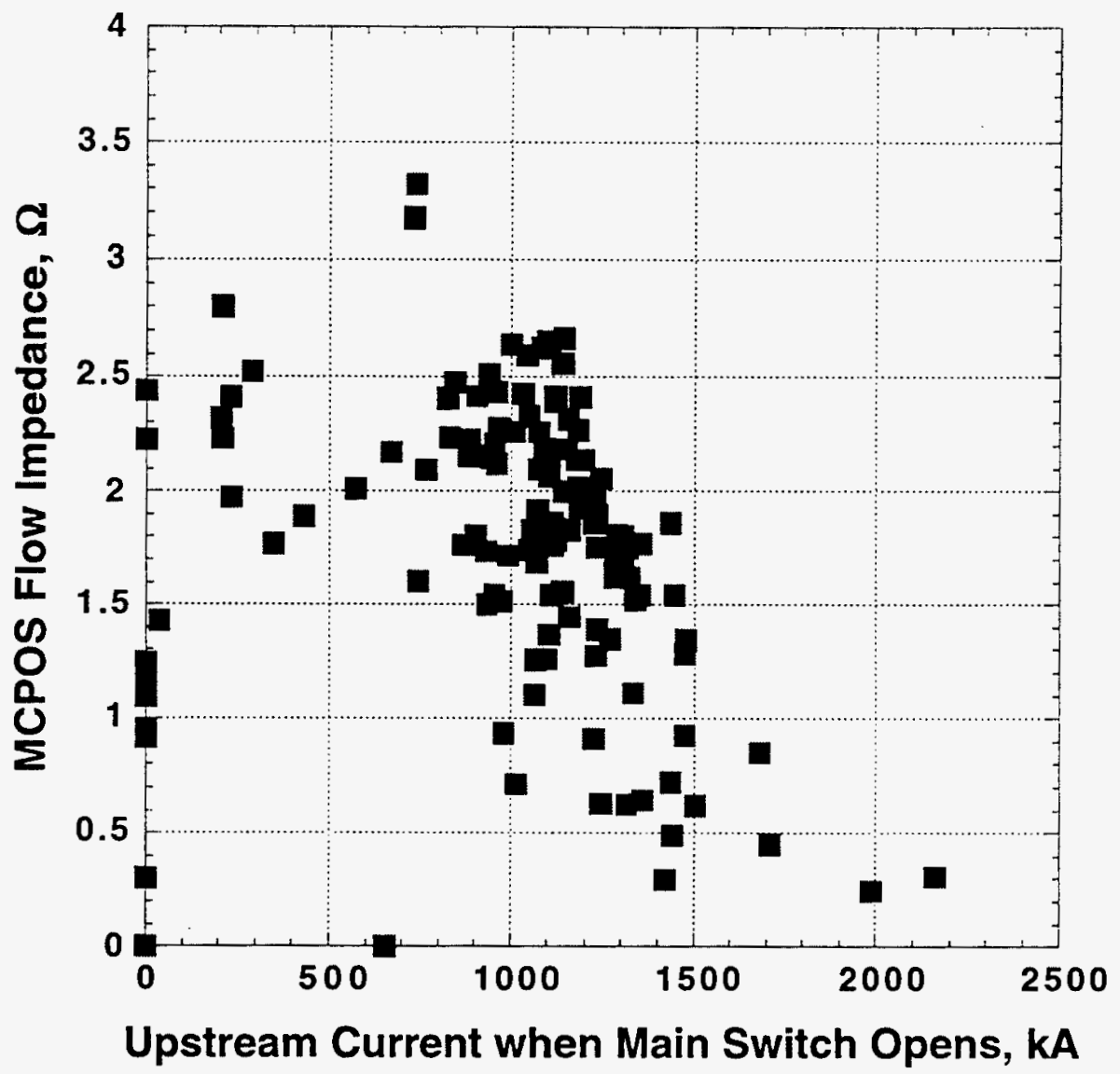

Figure 5-10. System flow impedance versus MCPOS opening current. The falling performance is due to the trigger switch. This is for all DM-2 experiments. 


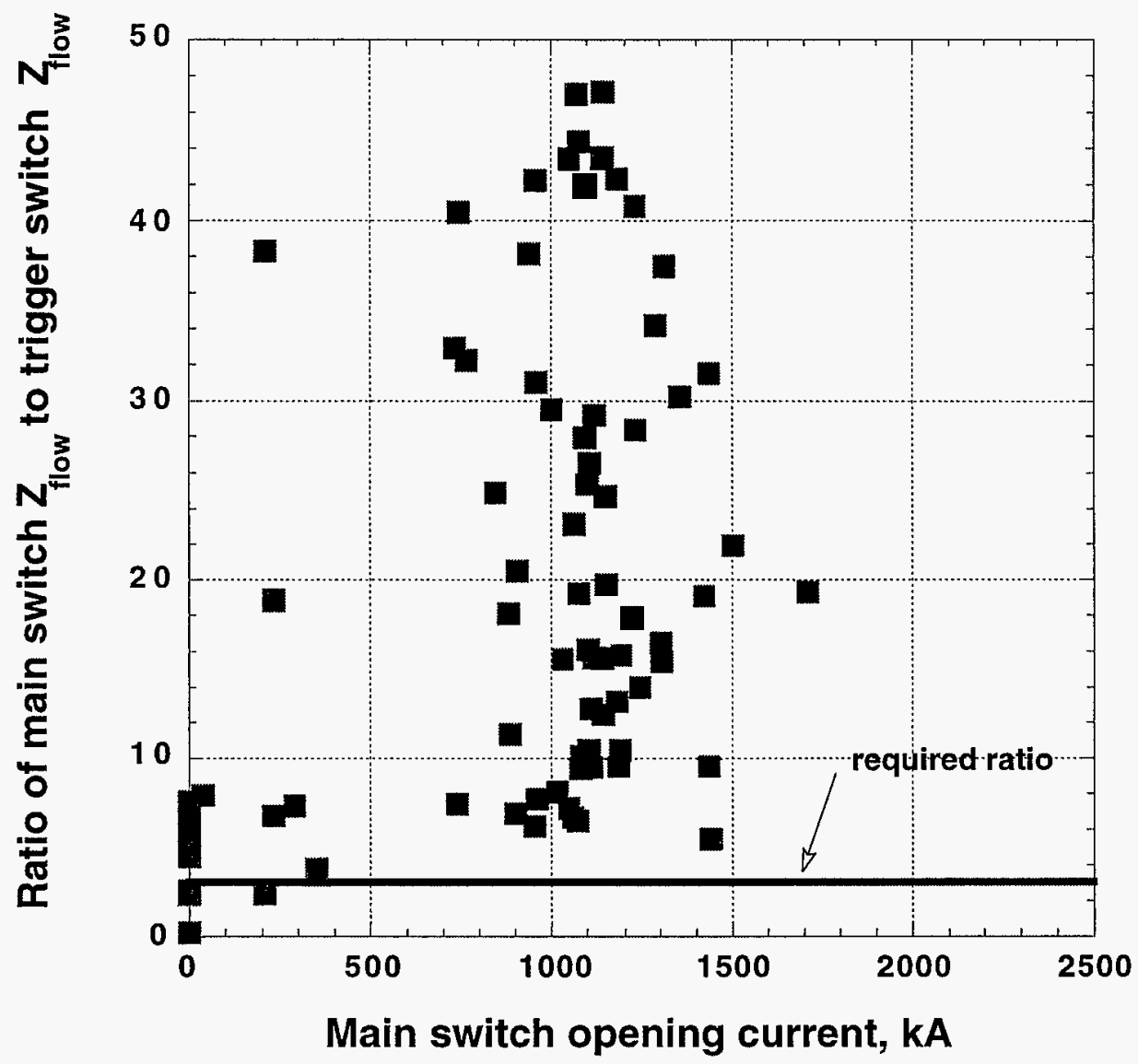

Figure 5-11. The ratio of the flow impedance of the main switch to the flow impedance of the trigger switch, at the time of peak main switch voltage. Also shown is the design requirement: trigger switch flow impedance of 1 $\Omega$ and MCPOS flow impedance of $3 \Omega$.

Figure 5-12 shows the MCPOS flow impedance on all DM-2 experiments, versus the ratio of applied magnetic field at the cathode to the axial magnetic field from the fast coil at the cathode. These data are taken at the time of peak switch voltage. As expected, tests with long plasma fill times (more plasma mass) lie below the static solution, and tests with less plasma mass lie above. The right side of the plot $\left(\frac{B_{\text {slow }}}{B_{\text {fast }}}=1.0\right)$ corresponds to an axial fast field at the cathode equal to the axial applied field at the cathode. Moving left requires either lowering the current in the applied field coil, or raising the current in the fast field coil. During a test, the applied field current is essentially un-changing, but the fast coil current rises when the trigger switch opens. Better trigger switch operation would move 
the operating point to the left. Also plotted on the Figure is the static magnetic field separatrix position. If the plasma boundary is approximated by the separatrix position, then the experimental values will lie near the static theory line. The plasma boundary can not in general be the same as the static separatrix location because of plasma incrtia and ion depletion from the plasma. Plasma inertia slows the motion of the plasma boundary when the opening starts. The force required to push the plasma armature depends upon the both the mass of plasma, and how fast the plasma is pushed by the magnetic field. A small mass pushed slowly would show less deviation from the static line than does a large mass or a fast acceleration, or both.

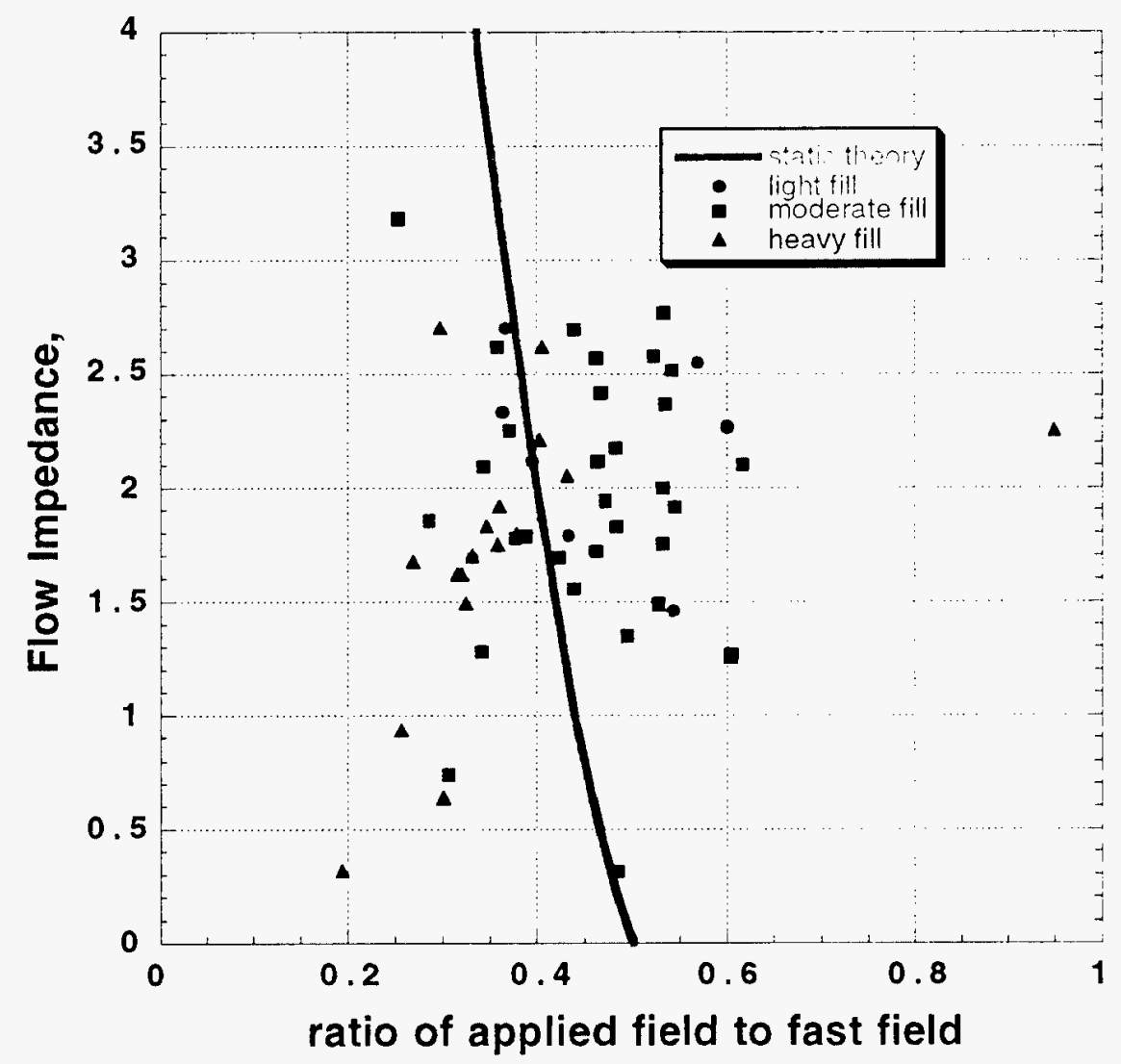

Figure 5-12. The flow impedance at peak power of the MCPOS as a function of the ratio of applied axial field to axial fast field at the cathode in the switch region. Light fill, moderate fill, and heavy fill refer to the plasma fill delay time in the MCPOS. Also plotted is the separatrix location (expressed in flow impedance) from the magnetostatic solution. 
Figure 5-13 shows the static separatrix position (in units of flow impedance) on a single DM-2 test, along with the flow impedance calculated from current and voltage monitors. The deviation from the low-mass theory line is due to plasma inertia. Plasma inertia can cause the actual opening to be less than the static theory predicts early in the pulse. Late in the pulse, inertia causes the opening to exceed the static prediction due to coasting of the plasma.

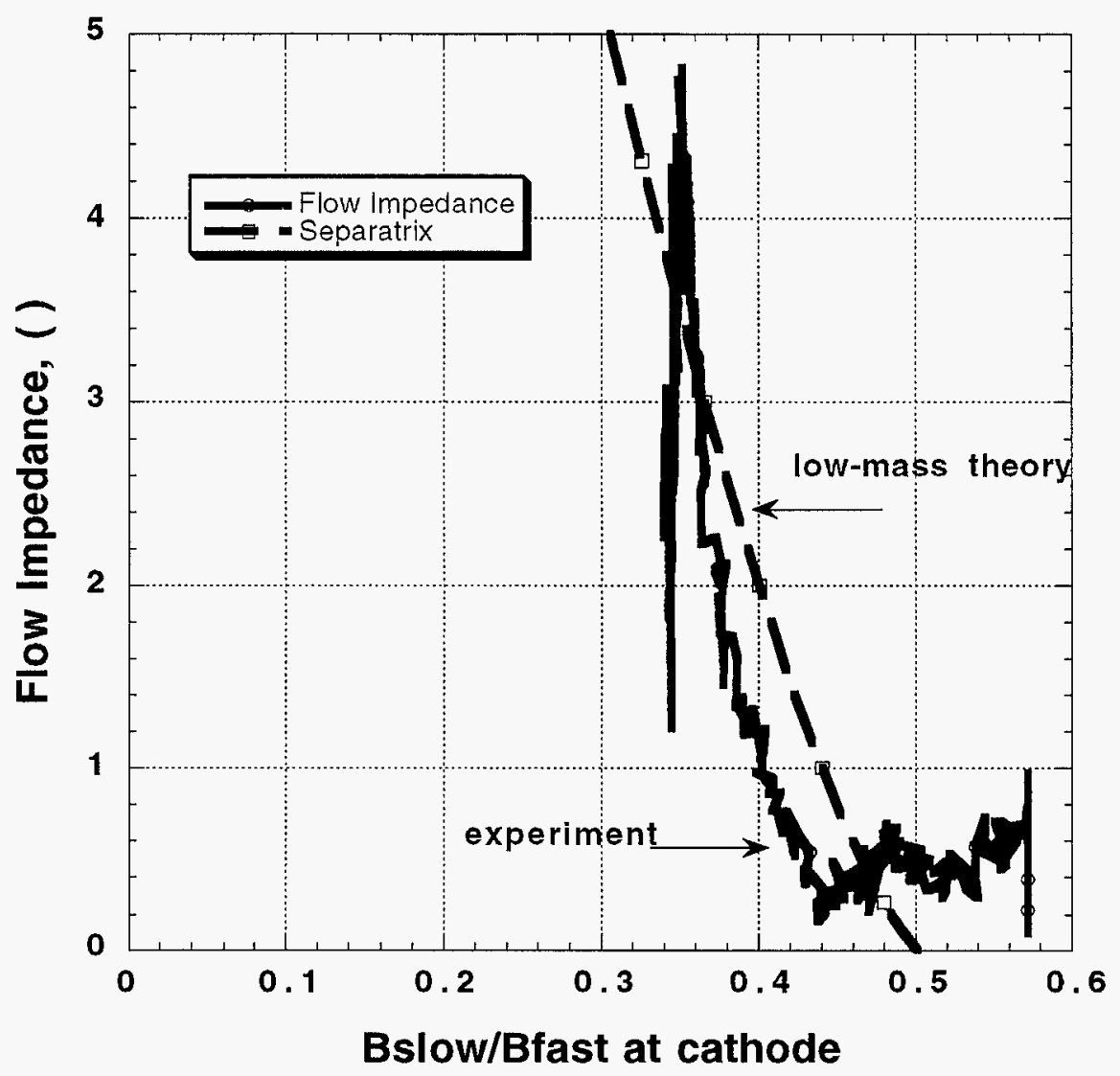

Figure 5-13. The flow impedance of the main switch on a single DM-2 test, versus the ratio of applied to fast field. Time progresses from left to right, as the fast axial field rises.

On this time scale (about $75 \mathrm{~ns}$ total) the applied field is unchanging. The agreement between the static model and the experiment shows this test had a small plasma mass, and that the plasma boundary generally agrees with the separatrix location. This would not happen if fast field flux penetrated the plasma. 


\section{Load design and performance}

Load impedance is important in any electrical system. Typically, terawatt particle beam loads have a falling impedance on a 50 ns time scale. ${ }^{54}$ This is due to surface plasma expansion and neutral charge-exchange, both of which act to close the effective gap. 55,56 Diodes at these power levels have electrode surfaces that are space-charge limited sources of ions and electrons. Gap closure happens faster at higher power densities. Figure 5-14 shows the load impedance obtained on a DM-2 test with the improved electron beam load (Figure 3-5 shows the DM-2 modified diode in cross-section). The impedance lifetime is defined to be $\tau_{z}=\frac{z}{\dot{z}}$, where $z$ is the impedance. Figure 5-14 shows an impedance lifetimc of about 75 ns.

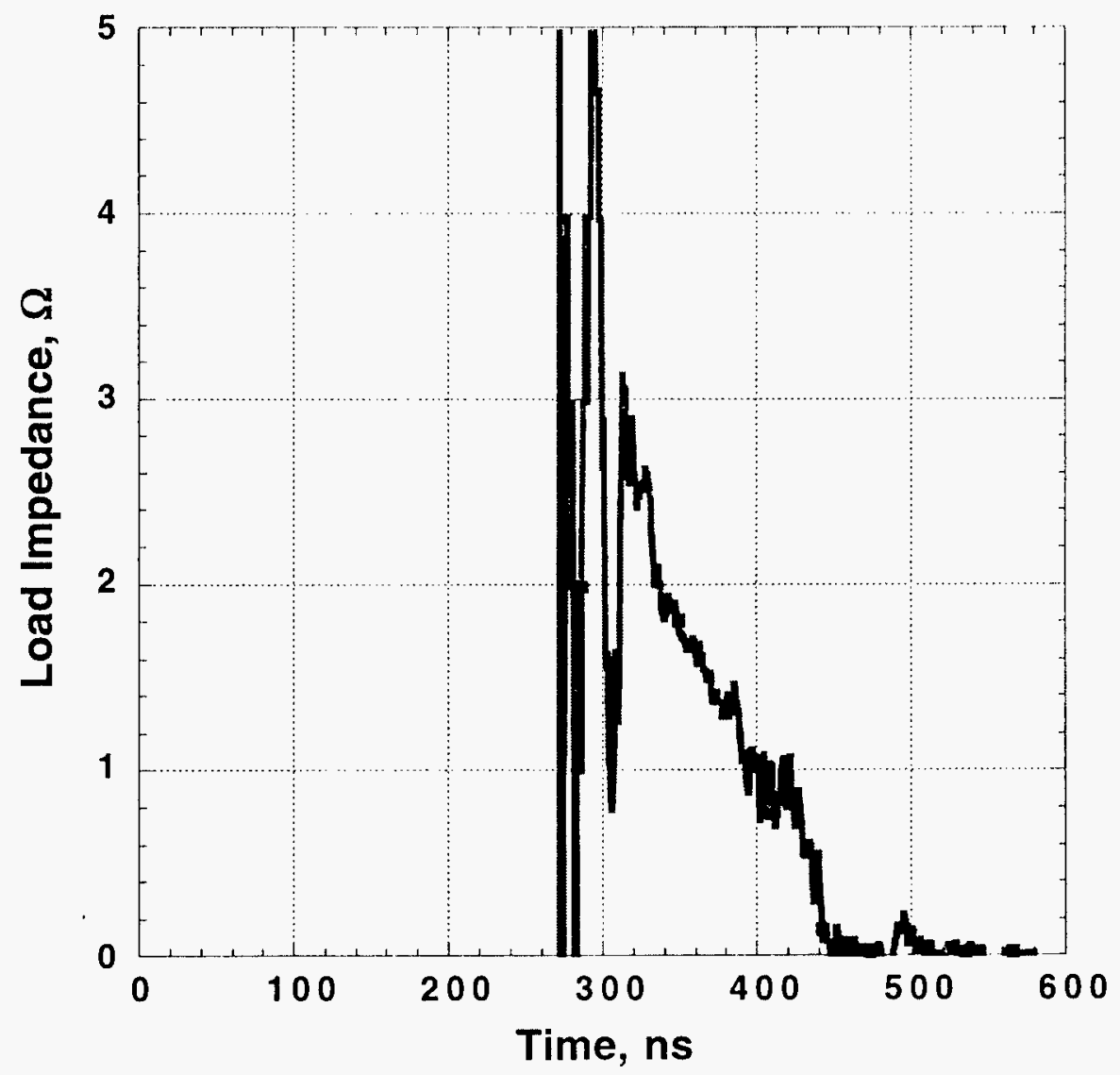

Figure 5-14. Load impedance on DM-2 shot 243. 
This diode has a second (bias) cathode inside the main cathode. This cathode could be independently adjusted. The diameter of the bias cathode was one-fourth that of the main cathode, so for equal gaps the bias current would be about one-fourth of the main cathode current. The modified load used on DM-2 had a better impedance lifetime because there was more active area, and therefore lower power density. The active area was larger because of both the increased cathode area and because the bias current drove electrons from the main cathode towards the center of the anode.

\section{Radiation production and measurements}

There was a total of ten DM-2 tests with a tantalum anode. On these tests, the Ta anode thickness was $0.1 \mathrm{~mm}$. There was also $0.8 \mathrm{~mm}$ of $\mathrm{Al}$ beam-stop, $10 \mathrm{~mm}$ of polyethylene, $5 \mathrm{~mm}$ polyamide debris shield, and a $0.25 \mathrm{~mm}$ mylar vacuum window. This large amount of beam-stop material was used to ensure that no electrons reached the dosimeters, as that would compromise those diagnostics. This was the configuration for all the radiation-measuring experiments on DM-2. There was no effort to optimize the converter/beam stop assembly, and modest dose gains could be made by using a smaller beam stop.

Unfortunately, the tantalum anode tests had several problems. First, the plasma contact electrode in the MCPOS that was changed at the end of the DM-2 tests changed the switch characteristics and so the plasma filltimes had to be re-optimized. There was not enough time in the few days to do an optimization. Second, during tantalum testing, the chamber had to be opened after every shot to change the tantalum anode. This may affect the electrode conditions in the main switch or trigger switch. Radio frequency (RF) glow cleaning 57 was used on some DM-2 tests in an attempt to reduce the effect of opening the chamber. The DM- 2 mechanical vacuum pump would not provide the $0.2 \mathrm{~Pa}$ pressures desirable, and in general there was insufficient time to investigate the effect of cleaning. The third problem was that the DM-2 Marxes were marginally reliable at $75 \mathrm{kV}$ charge level, and unreliable at $85 \mathrm{kV}$ charge. Evidently, the Marx trigger pulses were inadequate, and the Marx gas switches had to be operated at a very high fraction (about 80 percent, or more) of self-break voltage. Operation of the large number of switches in DM-2 at such a high fraction of self-break voltage inevitably causes prefires. So, careful optimization with the tantalum anode and the RF glow cleaning with the new plasma contact electrode was precluded. The electrical diagnostics showed that the few tantalum anode shots were inferior to many earlier $65 \mathrm{kV}$ stainless anode shots.

To quantify the effect of the tantalum versus stainless anodes, and to increase the amount of data available, the $\mathrm{X}$-ray production equation was used to find a scale constant for the PIN signal for both stainless and tantalum anodes. Although PI claimed that the lightly-filtered PIN signal on DM-1 changed little with stainless steel or tantalum anodes, 58 on the MCPOS experiments a clear and consistent factor of 3.3 times higher PIN diode 
signal with a tantalum anode was observed. This factor is a combination of scaling with converter atomic number, converter thickness, beam stop and vacuum chamber thickness. This scaling can be used to predict a PIN diode signal for either stainless anodes or Ta anodes. The correlation of the predicted PIN signal with the actual PIN signal is a verification of the accuracy of the load voltage and current.

The predicted PIN signal was calculated using the minimum of measured load anode current and measured anode current just downstream of the switch (to be as conservative as possible), and the load voltage extrapolated from the interface. The scaling with voltage assumed was $\beta=\mathbf{1 . 8}$. The calculated PIN signal to model a Ta anode is derived from

$$
P I N_{\text {calculated }}^{T a}=3.3 \bullet 10^{-16} I_{\text {load }}^{\text {anode }} V_{\text {load }}^{1.8}
$$

The PIN signal for a stainless steel anode could similarly be calculated from

$$
P I N_{\text {calculated }}^{\text {Stainless }}=1.0 \cdot 10^{-16} I_{\text {load }}^{\text {anode }} V_{\text {load }}^{1.8} \text {. }
$$

Figure 5-15 shows a plot of the predicted PIN signal versus the measured PIN signal for all the DM-2 shots with the Sandia data acquisition system. All the predicted PIN signals use the same scale constant, to predict the PIN signal for that electrical performance with a 0.004 inch $\mathrm{Ta}$ anode. In the plot, the line fitted to the Ta data (slope = 0.954; PIN predicted $\cong P I N_{\text {measured }}$ ) shows the accuracy of the PIN prediction; perfect agreement would have a slope of 1.0. The line fitted to the stainless steel data with slope 3.12 agrees with the ratio of PIN diode signals for Ta and stainless steel anodes $(3.12 / 0.954=3.3)$.

For reference, experiment 339, which had a Ta anode, has a measured dose of 12 kilorads DECADE equivalent. The twenty kilorad line on Figure 5-15 comes from scaling shot number 339 with the integral of the far-field PIN diode signal.

The fact that the stainless steel and Ta data accurately follow their respective lines shows two things: first, with an adequate experimental series, dose levels greater than 18 kilorads could be obtained; second, the corrected voltages are accurate. 


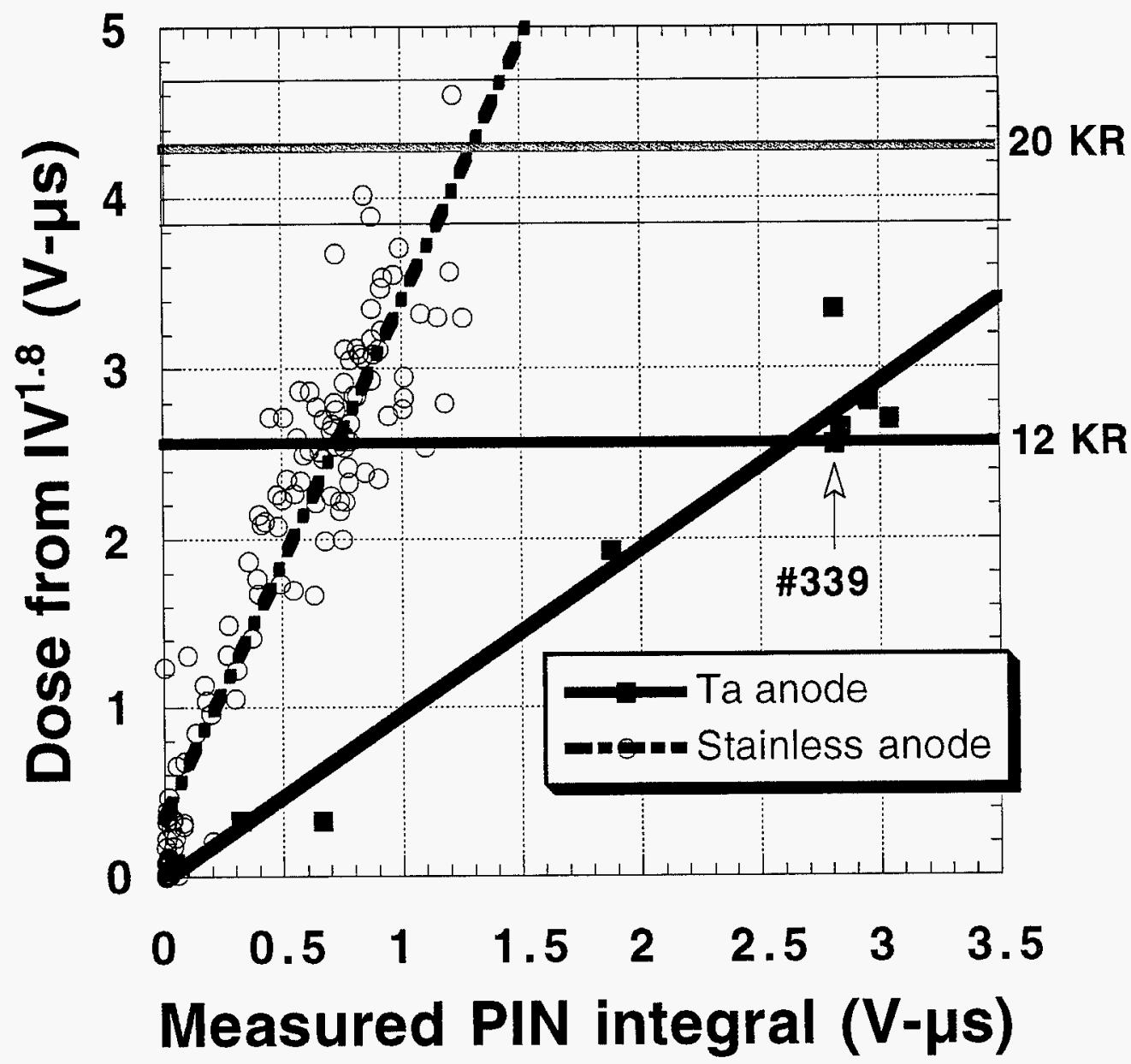

Figure 5-15. X-ray production from electrical diagnostics, and X-rays measured by the far-field PIN diode. The horizontal axis is the measured time-integral of the bare PIN diode; the vertical axis is the radiation signal calculated from load voltage and load current. 


\section{Discussion}

\section{Voltage monitors in the DM-2 system.}

Voltage measurements in inductive energy store experiments are both important and difficult. 42 Voltage measurements are critical to quantify opening switch performance and power flow. Because the voltage measurements are important, several tests were done periodically to assure the consistency of the data. One is to look at calculated load voltage on a test where load voltage is known. One case of known load voltage is a short circuit. Since the actual load voltage to zero, any non-zero load voltage calculated is a measure of error. Figure 6-1 shows the load voltage calculated on a short-circuit test. The error of $200 \mathrm{kV}$ is typical; this is within expected range for this initial series.

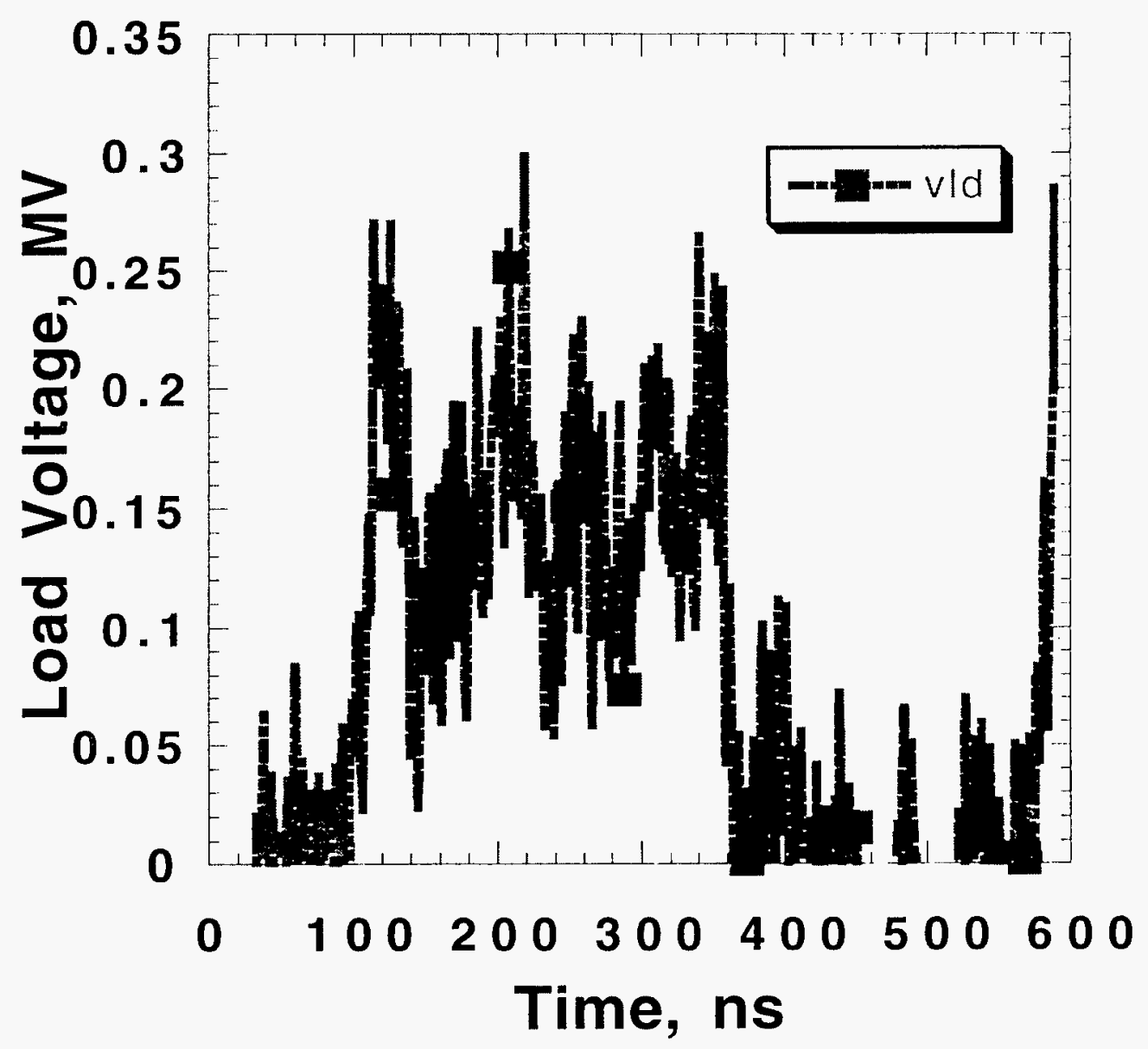

Figure 6-1. Load voltage extrapolated from the interface to the load on a test where the load was short-circuited. The actual load voltage is 
therefore zero; the indicated voltage demonstrates typical load voltage errors.

Figure 6-2 shows voltage at the MCPOS location corrected from both the interface and the load; on this test the load was a shorted so the load voltage was zero. Again, the error is about $200 \mathrm{kV}$.

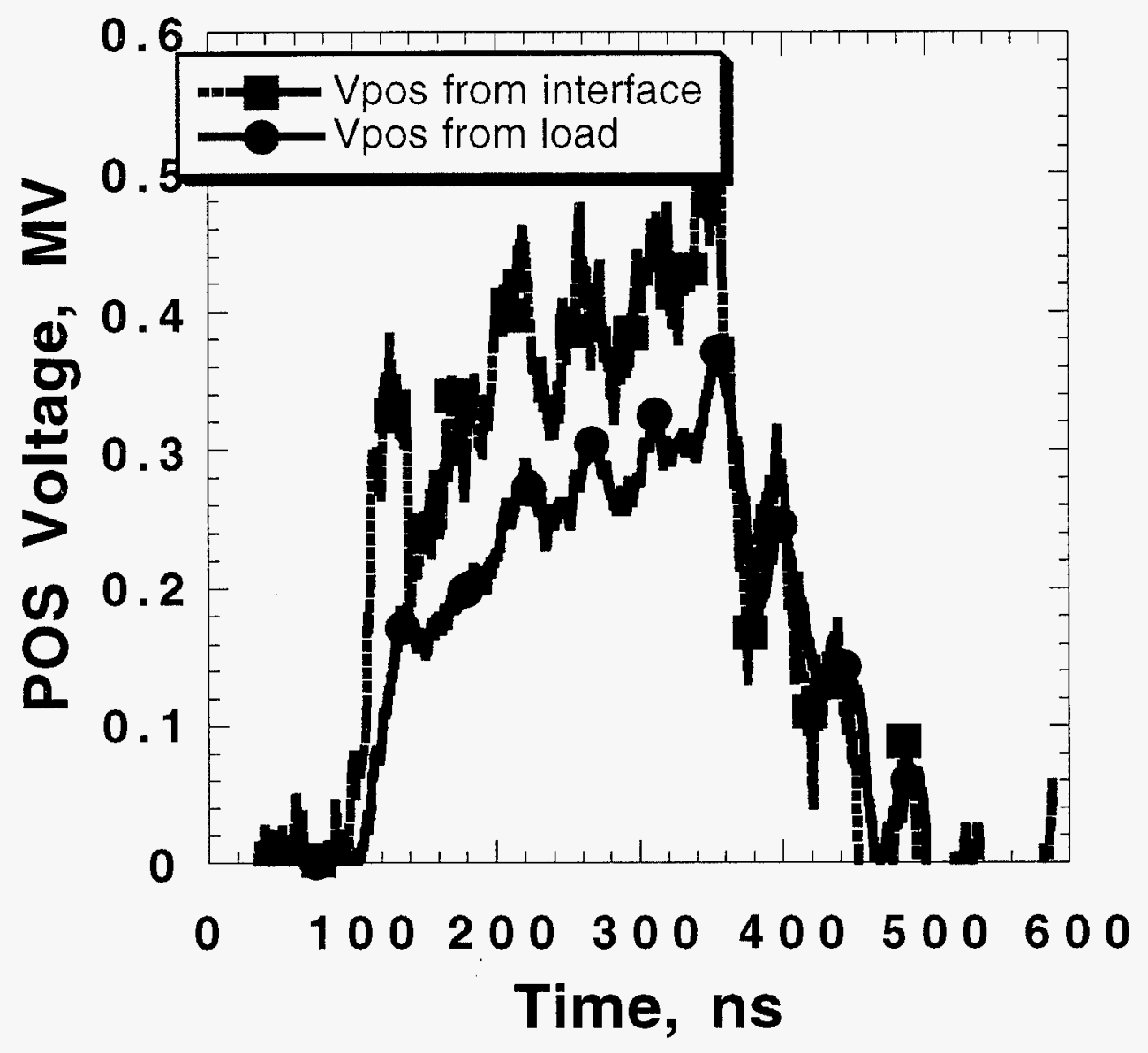

Figure 6-2. Opening switch voltage extrapolated from the interface to the switch, and from the load to the switch on a test with a short-circuit at the load.

Another voltage test involves the PIN diode. X-radiation reaching the detector varies as approximately voltage to the 1.8 power, and is linear with current. A plot of PIN signal versus calculated load voltage (Figure 6-3) gives an indication of voltage accuracy. The slope of the line in Figure 6-3 is 2.8; the accepted range for X-ray detection is 
$1.8 \leq \beta \leq 2.9$; unity is added to 1.8 to account for load current (proportional to $\mathrm{V}$ if the load impedance were constant).

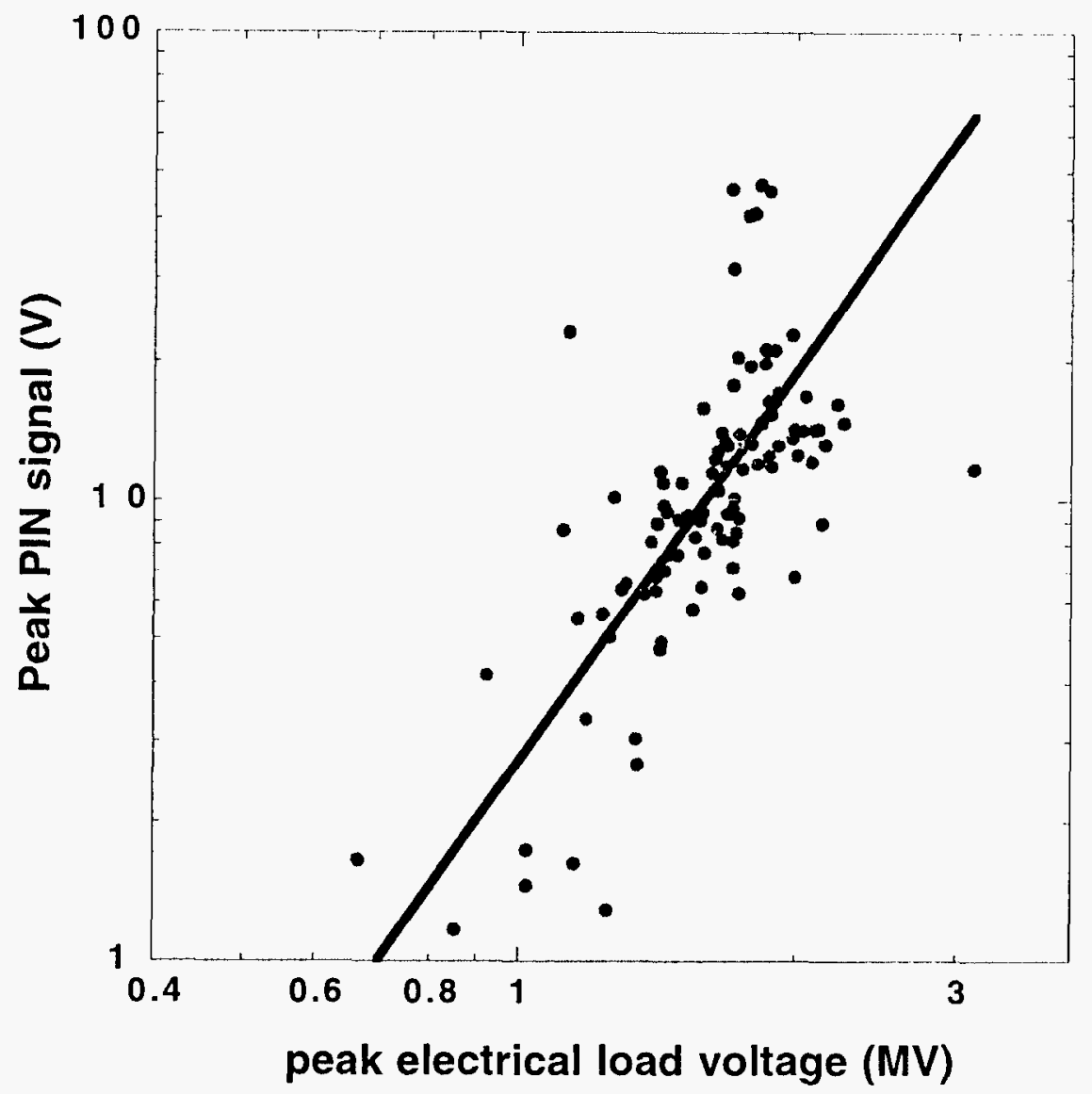

Figure 6-3. Peak voltage on the PIN diode signal vs. peak of the electrical load voltage. This shows all the stainless-steel anode tests, including 6 $\mathrm{mm}$ and $10 \mathrm{~mm}$ anode thickness.

A further test involves load voltage and current. By looking at several tests with Ta and stainless anodes, a model was developed for the PIN diode signal as discussed before. Figure 6-4 shows a comparison of the load voltage calculated from the interface voltage monitors, and the load voltage calculated from the far-field PIN diode. Solving the radiation production equation for voltage gives an equation for load voltage, as follows: 


$$
P I N=k \cdot I_{\text {load }} \cdot V_{\text {locd }}^{\beta}
$$

so

$$
V_{\text {load }}=\left(\frac{P I N}{k \cdot I_{\text {load }}}\right)^{\frac{1}{\beta}} \text {. }
$$

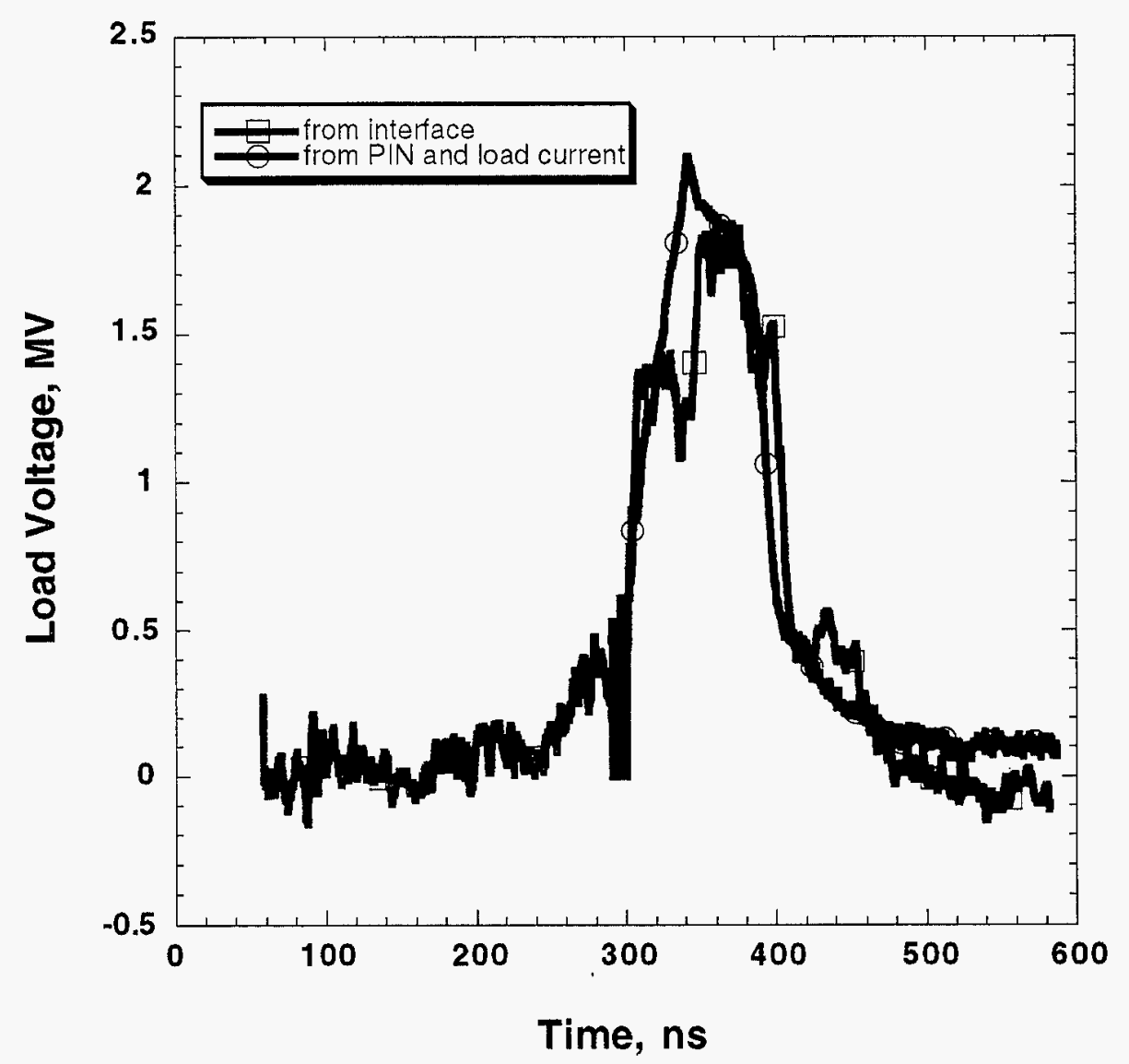

Figure 6-4. The electrical load voltage compared to a load voltage calculated from the PIN diode and the load anode current. This uses the same scaling constant as all DM-2 experiments. This was a Ta anode test.

The PIN diode signal is time-shifted back to the load location to account for timeof-flight. The scaling constant was the same as used for all DM-2 Ta anode experiments, which was 3.3 times higher than stainless anode experiments. For this electron beam system, load current is anode current. Using load anode current implies that all vacuumflowing electrons near the load contribute to radiation. The agreement between the voltage 
from dose-rate and electrical voltage shows that the monitors are consistent, and also shows that ions in the load region are insignificant, or at least a constant effect throughout the pulse. Attempts to do a similar calculation on a system with a MITL downstream of a conventional plasma opening switch have been unsuccessful. 59 


\section{Conclusions}

Sandia performed experiments with an advanced plasma opening switch, the magnetically controlled plasma opening switch. The MCPOS is a system designed to remove as much as possible the effects of variations in initial plasma fill conditions, and the effects of surface contaminants. This is done by using a magnetic field to hold the plasma in place for conduction, then another magnetic field to open the switch. The experiments proved that the MCPOS can be built in negative polarity as well as positive polarity. The MCPOS experiments showed the important result that this switch can drive low-impedance transmission lines downstream of the switch. This is vital for multi-modules drivers that are added together in vacuum, as is the case for all z-pinch loads.

There is a problem with the system on DM-2. This is the poor performance of the trigger switch. The trigger switch on DM-2 needed to work one-third as well as the main switch, and it did not. Part of the problem is due to plasma source asymmetries in the trigger switch plasma fill. This is a problem that could be due to faulty cables or capacitors. This would be the first area to investigate. Beyond that, there are some options for improving the trigger switch that would require some test time to validate the schemes. It is certainly preferable to develop a $1 \mathrm{TW}$ switch which can drive a proven $3 \mathrm{TW}$ switch, rather than doing trial-and-error development of a 3 TW switch. The driver energy required for trigger switch development is much smaller than required for main switch development, allowing testing on a smaller pulser. Further, the trigger switch is a stepping stone, as the ultimate interest is in a command-triggered opening switch. It is clear that activating the MCPOS with an external pulser will trigger opening. The fast coil is a lowimpedance load, and as such is difficult to drive; this is why the MCPOS experiments have used in inertial trigger POS. Still, the MCPOS is the only demonstrated TW plasma opening switch that has a viable trigger scheme developed.

The output of DM-2 with the MCPOS could also benefit from the addition of a load plasma fill at the brehmsstrahlung diode. This plasma filled load would have a more desirable load impedance temporal profile, and would reduce the effect of the inductance between the MCPOS and the load. With a $1.5 \Omega$ load and a $33 \mathrm{nH}$ MITL inductance, the load power pulse is appreciably affected by this inductance on a $20 \mathrm{~ns}$ time scale. A plasma-filled load would shorten the output pulse and raise the load voltage. Such a system would be effective because the MCPOS does not re-close, which would limit the output energy, and because of the short conduction time of the load plasma fill (about 20 ns).

With improvements to the trigger switch, and some other minor system changes, the MCPOS can be a reliable pulse compression element, for both electron-beam diode loads and fast $\mathrm{z}$-pinch loads. 


\section{References}

1P. Sincerney, K. Childers, J. Goyer et al., "DECADE design and testing status," presented at the Proceedings of the 11th International Conference on High Power Particle Beams, Prague, Czech Republic, 1996 (unpublished).

2P. J. Turchi, M. L. Alme, G. Bird et al., "Review of Plasma Flow Switch development," IEEE Trans. Plasma Sci. PS-15 (6), 747-759 (1987).

${ }^{3}$ Stanley Humphries, Jr., M. Savage, S. Coffey et al., "High-power openingclosing switches using grid-controlled plasmas," IEEE Trans. Plasma Sci. PS-13 (4), 177-187 (1985).

4J. M. Creedon, L.J. Demeter, B. Ecker et al., "The reflex switch: A new vacuum opening switch for use with magnetic energy storage," J. Appl. Phys. 57 (5), 1582-1588 (1985).

${ }^{5}$ C. W. Mendel, Jr. and S. A. Goldstein, "A fast-opening switch for use in REB diode experiments," J. Appl. Phys. 48 (3), 1004-1006 (1977).

6R. A. Meger, R. J. Commisso, G. Cooperstein et al., "Vacuum inductive store/pulse compression experiments on a high power accelerator using plasma opening switches," Appl. Phys. Lett. 42 (11), 943-945 (1983).

${ }^{7}$ R. W. Stinnett, D. H. McDaniel, G. E. Rochau et al., "Plasma opening switch development for the Particle Beam Fusion Accelerator II (PBFA II)," IEEE Trans. Plasma Sci. PS-15 (5), 557-563 (1987).

8"Special issue on fast opening vacuum switches," IEEE Trans. Plasma Sci. PS15 (6) (1987).

${ }^{9}$ R. Stringfield, R. Schneider, R. D. Genuario et al., "Plasma erosion switches with imploding plasma loads on a multiterawatt pulsed power generator," J. Appl. Phys. 52 (3), 1278-1284 (1981).

${ }^{10}$ H. Bluhm, K. Böhnel, P. Hoppé et al., "A summary of experimental results from inductive store pulse compression experiments with plasma opening switches on the pulse power generators Pollux and KALIF," IEEE Trans. Plasma Sci. PS-15 (6), 654666 (1987).

11 A. Ron, A.A. Mondelli, and N. Rostoker, "Equilibria for magnetic insulation," IEEE Trans. Plasma Sci. PS-1, 85 (1973).

12 John M. Creedon, "Relativistic Brillouin flow in the high $v / \gamma$ diode," J. Appl. Phys. 46 (7), 2946-2955 (1975). 
${ }^{13}$ E. I. Baranchikov, A. V. Gordeev, V. D. Korolev, et al., "Magnetic selfinsulation of electron beams in vacuum lines," Sov. Phys. JETP 48 (6), 1058-1068 (1979).

$14 \mathrm{~J} . \mathrm{P}$. Vandevender, "Long self-magnetically insulated power transport experiments," J. Appl. Phys. 50 (6), 3928-3934 (1979).

${ }^{15}$ C. W. Mendel, Jr., D. B. Seidel, and S. E. Rosenthal, "A simple theory of magnetic insulation from basic physical considerations," Laser and Particle Beams 1 (3), 311-320 (1983).

${ }^{16}$ C. W. Mendel, Jr., D. B. Seidel, and S. A. Slutz, "A general theory of magnetically insulated electron flow," Phys. Fluids 26 (12), 3628-3635 (1983).

${ }^{17}$ C. W. Mendel, Jr., G. E. Rochau, M. A. Sweeney et al., "Launched Electrons in Plasma Opening Switches," presented at the Novosibirsk Opening Switch Workshop, Novobirsk, Siberia, USSR, 1989 (unpublished).

18B. V. Weber, J. R. Boller, R. J. Commisso et al., "Microsecond-conductiontime POS experiments," presented at the 9th International Conference on High-Power Particle Beams, Washington, D.C., 1992 (unpublished).

19Donald E. Parks and Paul Steen, "Circuit characterization of magnetically insulated electron flow," presented at the 10th International Conference on High Power Particle Beams, San Diego, California, 1994 (unpublished).

20 S. B. Swanekamp, J. M. Grossman, P. F. Ottinger et al., "Power flow between a plasma-opening switch and a load separated by a high-inductance magnetically insulated transmission line," J. Appl. Phys. 76 (5), 2648-2656 (1994).

21 L. E. Aranchuk, A. S. Chuvatin, and A. V. Gordeev, "Model for calculation of output characteristics of nanosecond REB generator with plasma opening switch," presented at the Eighth International Conference on High-Power Particle Beams, Novosibirsk, USSR, 1990 (unpublished).

22Alexander Chuvatin, "Etude des Phases de Conduction et d'Ouverture d'un Commutateur à Plasma,", Ecole Polytechnique, 1993.

${ }^{23}$ L. E. Aranchuk, V. B. Babykin, A. S. Chernenko et al., "Passage of an intense electromagnetic pulse in a vacuum line with a plasma switch," Sov. J. Plasma Phys. 17 (5), 317-322 (1991).

${ }^{24}$ V. M. Bystritskii and I. V. Lisitsyn, "Experimental studies of a microsecond plasma opening switch in the positive polarity regime," presented at the 9th International Conference on High-Power Particle Beams, Washington, D.C., 1992 (unpublished). 
25J.R. Goyer, D. Kortbowi, F.K. Childers et al., "Opening Switch Research and Development for DECADE," presented at the 10 International Conference on High Power Particle Beams, San Diego, California, 1994 (unpublished).

26P. J. Goodrich, R. J. Commisso, J. M. Grossman et al., "High power plasma opening switch operation on HAWK," presented at the 10th International Conference on High Power Particle Beams, San Diego, California, 1994 (unpublished).

${ }^{27}$ C. W. Mendel, Jr., M. E. Savage, D. M. Zagar et al., "Experiments on a current-toggled plasma-opening switch," J. Appl. Phys. 71 (8), 3731-3746 (1992).

28 M.E. Savage, E.R. Hong, W.W. Simpson et al., "Plasma Opening Switch Experiments at Sandia National Laboratories," presented at the 10th International Conference on High Power Particle Beams, San Diego, California, 1994 (unpublished).

${ }^{29}$ M. E. Savage, W. W. Simpson, G. W. Cooper et al., "Long conduction time plasma opening switch experiments at Sandia National Laboratories," presented at the 9th International Conference on High-Power Particle Beams, Washington, D.C., 1992 (unpublished).

30 G. S. Mills, "Optimization of a high density, distributed plasma source," Bull. Amer. Phys. Soc. 26, 988 (1981).

31 J. R. Woodworth, E. J. T. Burns, D. L. Hanson et al., "Surface discharges for soft X-ray preionization in Light-Ion-Fusion accelerators," IEEE Trans. Plasma Sci. PS15 (4), 384-393 (1987).

${ }^{32}$ C. W. Mendel, Jr., M. E. Savage, D. M. Zagar et al., "Carbon $4+$ Plasma Source," presented at the 1990 IEEE International Conference on Plasma Science, Oakland, Ca., 1990 (unpublished).

${ }^{33}$ H. J. Doucet, J. M. Buzzi, M. Gazaix et al., "Basic plasma physics research in pulsed-power technology at Ecole Polytechnique," Laser and Particle Beams 5 (3), 451456 (1987).

${ }^{34}$ S. Humphries, Jr., R. J. M. Anderson, J. R. Freeman et al., "Pulsed plasma guns for intense ion beam injectors," Rev. Sci. Instrum. 52 (2), 162-171 (1981).

${ }^{35}$ C. W. Mendel, Jr., D. M. Zagar, G. S. Mills et al., "Carbon plasma gun," Rev. Sci. Instrum. 51 (12), 1641-1644 (1980).

36 Stanley Humphries, Jr., Charged Particle Beams (Wiley, New York, 1990).

${ }^{37}$ C. W. Mendel, Jr., "Conical Z-pinch gun," J. Appl. Phys. 42 (13), 5483-5491 (1971). 
${ }^{38}$ N. R. Pereira and J. Davis, " $\mathrm{X}$ rays from z-pinches on relativistic electron-beam generators," J. Appl. Phys. 64 (3), R1-R27 (1988).

${ }^{39}$ T. W. L. Sanford, T. J. Nash, R. C. Mock et al., "Dynamics of a high-power aluminum-wire array Z-pinch implosion," Phys. Plasmas 4 (6), 2188-2203 (1997).

40 D. B. Seidel, private communication.

${ }^{41}$ C. W. Mendel, Jr. and S. E. Rosenthal, "Modeling magnetically insulated devices using flow impedance," Phys. Plasmas 2 (4), 1332-1342 (1995).

${ }^{42}$ M. E. Savage, Jr. C. W. Mendel, T. W. Grasser et al., "Time-resolved voltage measurements in terawatt magnetically insulated transmission lines," Rev. Sci. Instrum. 61 (12), 3812-3820 (1990).

${ }^{43}$ C. W. Mendel, Jr., Internal memorandum Report No. RS 4244/80/2, 1980.

${ }^{44}$ Robert W. Kuckuck, Report No. UCRL-51011.

${ }^{45}$ Frank H. Attix, Introduction to Radiological Physics and Radiation Dosimetry (Wiley, 1986).

${ }^{46} \mathrm{H}$. W. Koch and J. W. Motz, "Bremsstrahlung cross-section formulas and related data," Rev. Mod. Phys. 31 (4), 920-955 (1959).

${ }^{47}$ North Star Research, FR series integrators.

${ }^{48}$ S Shope, J.W. Poukey, K.D. Bergeron et al., "Self-magnetic insulation in vacuum for coaxial geometry," J. Appl. Phys. 49 (7), 3675-3678 (1978).

49 John M. Creedon, "Magnetic cutoff in high-current diodes," J. Appl. Phys. 48 (3), 1070-1077 (1977).

50L. E. Aranchuk, E. I. Baranchikov, A. V. Gordeev et al., "Magnetically selfinsulated lines with ion leaks," Sov. Phys. Tech. Phys. 34 (2), 215 (1989).

${ }^{51} \mathrm{C} . \mathrm{W}$. Mendel, Jr. and S.E. Rosenthal, "Dynamic modeling of magnetically insulated transmission line systems," Phys. Plasmas 3 (11), 42074219 (1996).

52Mark E. Savage, "Experimental studies of self-magnetically insulated relativistic electron flows,", University of New Mexico, 1989.

${ }^{53}$ C. W. Mendel, Jr., "15-cm hybrid ion diode on PBFA-I," J. Appl. Phys. 62 (9), 3522-3534 (1987). 
54J. E. Maenchen, J. R. Woodworth, T. A. Mehlhorn et al., "Extreme-ultraviolet illumination effects on the PBFA-I magnetically insulated ion diode," J. Appl. Phys. 65 (2), 448-463 (1988).

${ }^{55}$ T. W. L. Sanford, J. A. Halbleib, J. W. Poukey et al., "Measurement of electron energy deposition necessary to form an anode plasma in $\mathrm{Ta}, \mathrm{Ti}$, and $\mathrm{C}$ for coaxial bremsstrahlung diodes," J. Appl. Phys. 66 (1), 10-22 (1989).

56D. W. Swain, S. A. Goldstein, L. P. Mix et al., "The characteristics of a medium current relativistic electron-beam diode," J. Appl. Phys. 48 (3), 1085-1093 (1977).

57 Michael E. Cuneo, Peter R. Menge, David L. Hanson et al., "Results of vacuum cleaning techniques on the performance of $\mathrm{LiF}$ field-threshold ion sources on extraction applied-B ion diodes at 1-10 TW," IEEE Trans. Plas. Science 25 (2), 229-251 (1997).

${ }^{58}$ D. Kortbawi, private communication.

${ }^{59}$ David Kortbawi, F. K. Childers, J. M. Creedon et al., "Experiments on a long magnetically insulated transmission line downstream of a PEOS," presented at the 8th IEEE International Pulsed Power Conference, San Diego, 1991 (unpublished). 
DISTRIBUTION :

2 Numerex

Attn: Mike Frese (1) Jack Waltrous (1)

2309 Renard PI. S.E. Suite 220

Albuquerque, NM 87106

2 Berkeley Research Associates

Attn: Nino Pereira (1)

Douglas Weidenheimer (1)

PO Box 852

Springfield, VA 22150

1 North Star Research, Inc.

Attw: Richard Adler

4421 Mcleod Rd., N.E.

Suite A

Albuquerque, NM 87109

1 Logicon RDA

Attn: Ihor Vitkovitsky 2100 Washington Blvd.

Arlington, VA. 22204

6 Maxwell Technologies, Inc.

Federal Division

Attn: John Thompson (1)

John Goyer (1)

William Rix (1)

Paul Steen (1)

Don Parks (1)

Karin Robertson (1)

8888 Balboa Ave.

San Diego, CA 92123

3 DSWA

Attn: Ralph Schneider (3)

6801 Telegraph Rd.

Alexandria. VA 22310

4 Primex Physics International

Attn: David Kortbawi (1)

Peter Sincerney (1)

Jerry Levine (1)

Terry Tucker (1)

2700 Merced St.

PO Box 5010

San Leandro, CA 94577-0599

2 Pulse Sciences, Inc.

Attn: Phil Spence (1)

Sid Putnam (1)

600 McCormick Street

San Leandro, CA 94577
3 Pulsed Power Physics Branch,

Plasma Physics Division

Attn: Bruce Weber (1)

Robert Commisso (1)

Gerald Cooperstein (1)

Naval Research Laboratory

4555 Overlook Ave., S.W.

Washington, D.C. 20375-5346

1

Alameda Applied Sciences, Inc.

Attn: Mahadevan Krishnan

2235 Polvorosa Ave.

Suite 230

San Leandro, CA 94577

Arnold Engineering Development

Center

Attr: Jed Rowley

Arnold Air Force Base, Tenn. 37389

MS 1152 Leonard Martinez, 9543

1152 Larry Schncider, 9543

1178 Juan Ramirez. 9580

1182 Tim Renk. 9514

1182 Doug Bloomquish 9530

1184 John Boyes, 9539

1184 David L. Johnson. 9539

1184 Bill Shoup, 9539

1186 Paul Mix, 9542

1186 David Seidel, 9542

1186 Michael Cunco, 9533

1187 Keith Matzen. 9570

1190 Donald Cook. 9500

1191 Jeff Quintenz, 9502

1193 John Maenchen, 9515

1194 Dillon McDaniel , 9540

1194 Mark Savage, 9543

1194 Chris Deency, 9544

1194 Tom Martin, 9544

1194 Clifford Mendel, 9544

1194 Ken Prestwich, 9544

1194 Johann Seamen, 9544

1194 Rick Spielman, 9544

1194 Ken Struve, 9544

1194 Walter Simpson, 9572

9018 Central Technical

Files, 8940-2

0899 Technical Library, 4916

0619 Review \& Approval

Desk, 12690

for DOE/OSTI 


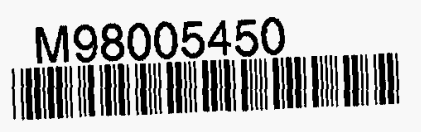

Report Number (14) SAND - $98-0704$

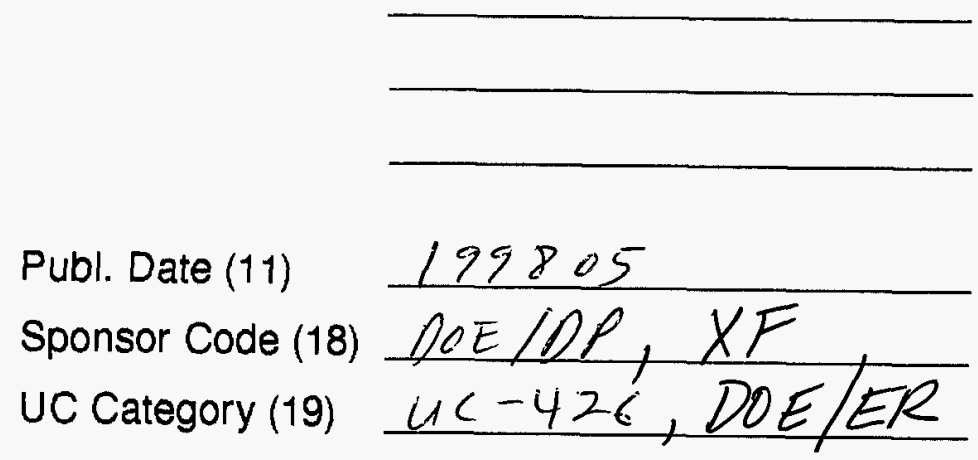

\section{3}

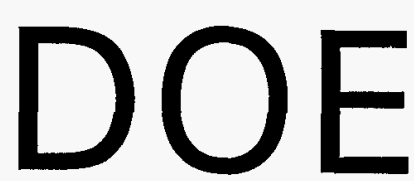

